\title{
Modelling of acoustic and elastic wave propagation from underground structures using a 2.5D BEM-FEM approach
}

\author{
A. Romero ${ }^{1}$, P. Galvín ${ }^{1}$, J. António ${ }^{2}$, J. Domínguez ${ }^{1}$, A. Tadeu ${ }^{2}$ \\ ${ }^{1}$ Escuela Técnica Superior de Ingeniería, Universidad de Sevilla, Camino de los Descubrimientos, 41092 Sevilla, Spain \\ ${ }^{2}$ Department of Civil Engineering, University of Coimbra, Pólo II, Rua Luís Reis Santos, 3030-788 Coimbra, Portugal
}

\begin{abstract}
This paper presents a numerical method based on a two-and-a-half dimensional (2.5D) boundary elementfinite element (BEM-FEM) coupled formulation to study noise and vibration from underground structures. The proposed model properly represents the soil-structure interaction problem and the radiated noise and vibration. The soil is modelled with the boundary element method, and the Green's function for a fluid-solid formation is taken as the fundamental solution to represent a solid half-space flattened by a fluid medium, which represents the soil and the air above the ground surface. The finite element method is used to represent structures and enclosed air volumes. The problem representation is limited to a soil-structure interface and the ground surface does not need to be discretised. Radiated noise and vibration are determined after the soil-structure interaction problem has been solved. We verify the proposed method by comparing the solution with an analytical solution for the wave propagation in a fluid-solid medium. Three examples are given to illustrate the noise and vibration radiated by tunnels. The results show that the soil-structure interaction influences the sound pressure field above the ground surface.
\end{abstract}

Keywords: 2.5D BEM-FEM, noise and vibration, tunnels, soil-structure interaction, fluid-structure interaction

\section{Introduction}

In their efforts to achieve sustainable growth many countries have implemented an efficient transportation model to separate economic growth from the use of resources, with lower use of fuel and carbon based systems. Several areas have therefore been modernised, including connected urban and interurban train, metro, and tram systems. However, noise and vibration are among the most common environmental impacts of mass transit solutions, which stem from different sources [1]. Transportation noise can indeed cause annoyance by disrupting sleep, interfering with communication, adversely affecting health and even adversely affecting academic performance. Some studies on general annoyance and activity disturbances show that

Email address: aro@us.es (A. Romero ${ }^{1}$ ) 
train noise is more of a nuisance when there is simultaneous exposure to vibration. This is because it is difficult to distinguish between noise and vibration and this exacerbates the annoyance from noise $\underline{[2,3]}$. Because transport noise pollution affects large numbers of people, railway infrastructure managers require an assessment of the disturbances caused by transport operation and their mitigation.

Ground-borne vibration and radiated noise from underground traffic is another major environmental concern in urban areas. The vibration propagates through the underground structure and the surrounding soil and it is perceived directly and sensed indirectly as radiated noise. Technically-supported decisions should be backed up by the accurate numerical modelling of acoustic and elastic waves.

The BEM is well suited to represent unbounded problems and the discretization is limited to the boundary. The influence matrices of the BEM are fully-populated and its computation would involve a high computational effort when the problem size is large. Some authors have proposed enhancements based on the fast multipole expansion method (FMM) [4, 5] and on the accelerated BEM by the precorrected fast Fourier transform method (pFFT) [6]. The accelerated BEM based on hierarchical matrices $\mathcal{H}$ and $\mathcal{H}^{2}$ allows the matrix-vector multiplication with almost linear complexity $\underline{[7,8]}$. Brunner et al. $\underline{[9]}$ compared the BEM based either on the FFM and the hierarchical matrices for the Helmoltz problem, concluding that the computation effort for matrix-vector products was significantly lower when using the hierarchical matrices. Messner and Schanz $\underline{[10]}$ used $\mathcal{H}$-matrices in the development of an accelerated time-domain BEM for elasticity. These authors separated the far-field from the near-field by means of the hierarchical matrices. They improved the compression of the BEM matrices by separating into a sum of $\mathcal{H}$ and $\mathcal{H}^{2}$ matrices. The accelerated methodologies reduce the computational effort in order to represent three-dimensional problems but the $2.5 \mathrm{D}$ formulation is preferred to study quite long invariant geometries. For this, several numerical models based on two-and-a-half dimensional (2.5D) formulations have been proposed to account for the longitudinally invariant geometry of structures such as tunnels.

These formulations compute the three-dimensional (3D) wave field from two-dimensional (2D) problems with different wavenumbers [11] to avoid the computationally expensive disadvantage of 3D formulations. Forrest and Hunt $[12]$ are among the authors who have proposed a semi-analytical solution for the timeharmonic displacements of a tunnel modelled as an infinitely long, thin cylindrical shell, where the soil was represented as a homogeneous full-space. The coupled problem was solved in the frequency domain by Fourier decomposition into ring modes circumferentially and a Fourier transform into the wavenumber domain longitudinally. François et al. [13] presented a 2.5D coupled BEM-FEM methodology to compute the dynamic interaction between a layered soil and structures with longitudinally invariant geometry. This formulation uses a 2.5D Green's function for a layered half-space, thus there is no need to discretise the free surface and the layer interfaces. A regularised boundary integral equation is derived to avoid the evaluation of singular traction integrals. Later, Galvín et al. $\underline{[14]}$ used that formulation to predict railway induced vibrations in a tunnel embedded in a layered half-space. Moreover, Gupta et al. [15] proposed a coupled 
periodic BEM-FEM approach, where a boundary element method is used for the soil and a finite element method for the tunnel. The tunnel periodicity is handled by the Floquet transformation to formulate the tunnel-soil interaction problem in the frequency-wavenumber domain, and to compute the wave propagation field in the soil. Furthermore, a windowing technique was proposed by Coulier et al. [16] to represent finite domain dimensions in a 2.5D formulation to study SSI problems. The proposed method allowed the study of finite insulation trenches within an invariant soil geometry.

Many works suggest radiated noise should be computed assuming that the sound receiver has no effect on the vibration generation mechanism. Therefore, this simplification allows the decoupling of the incident wave field and the radiated noise. Following this procedure, Nagy et al. [17] proposed a Rayleigh integral-based method combined with the FEM to predict radiated noise in buildings from underground railway traffic. This model was experimentally validated in a building close to a railway line in Paris and maximum sound pressure level was found to be $75 \mathrm{~dB}$. Fiala et al. [18] also suggested a numerical model to study vibrations and radiated noise caused by underground railways. This model solves the soil-structure interaction problem with a BEM-FEM coupled formulation using the Green's function for the layered half-space. The acoustic radiation problem is then computed assuming weak coupling between structural and acoustic waves. An acoustic spectral finite element method is used to predict the radiated noise. Tadeu et al. [19] subsequently modelled the acoustic attenuation provided by a barrier in an underground train station using a comprehensive coupled formulation based on the BEM and the method of fundamental solutions (MFS). In this case, the proposed method can compute the noise without the former simplifications. Recent publications have produced computationally feasible numerical models which can represent the ground-borne vibration at specific sites. Romero et al. [20] presented a 2.5D BEM-FEM model to study noise and vibration within a tunnel embedded in an unbounded solid due to a moving load. They used the BEM for the full-space fundamental solution in elastodynamic and the FEM in fluid-acoustics and elastodynamics. The results showed that the tunnel displacement and the air pressure inside the tunnel increase with the load speed according to the regime defined by the wave propagation velocity in each medium. The same behaviour was observed in the unbounded solid. However, to the best of our knowledge the radiated noise and vibration at the soil surface from underground structures has not been studied using a coupled formulation.

This work describes a novel numerical model that can handle the above mentioned problem. The model takes a domain decomposition approach to study fluid acoustics and solid scattering waves in a half-space medium with an innovative BEM technique. The BEM formulation considers, as fundamental solution, a problem where a formation composed of an elastic solid medium bounded by an acoustic fluid medium that can be the soil as well the air above the ground surface. Therefore, the discretisation of the fluid-solid interface is not needed and only soil-structure interfaces are modelled. The proposed methodology is used to study the acoustic and elastic scattered wave field inside underground structures, within the soil, and above the ground surface. The paper is organised as follows. First, the numerical model is presented, the 2.5D 
BEM-FEM fluid-acoustics formulation is described, and the Green's function for a solid half-space bounded by a fluid formation is discussed. The proposed method is then verified with a benchmark problem that studies the wave propagation in fluid-solid formations. This section also analyses the convergence of the longitudinally spatial Fourier transform. The proposed method is then used to study noise and vibration in three examples related to the wave field radiated by a single tunnel, a twin tunnel, and an underground station with a sound insulation screen. Finally, the conclusion section summarises the main contributions of this work.

\section{Numerical model}

The numerical model is based on a coupled BEM-FEM formulation (Figure 1). The underground structure, composed by solid $\left(\Omega_{s}\right)$ and fluid $\left(\Omega_{f}\right)$ enclosures, is represented by the FEM. The interface between both enclosures is denoted as $\Gamma_{q}$. The soil domain $\left(\Omega_{s \infty}\right)$ and the air above the ground surface $\left(\Omega_{f \infty}\right)$ are described by the BEM. The Green's function for a fluid-solid formation presented by Tadeu and António $\underline{21]}$ is used as fundamental solution in the BEM. Therefore, the boundary element discretisation is limited to the limiting interface between the soil and the structure $\left(\Gamma_{o}\right)$. The coupled BEM-FEM formulation is addressed by imposing proper conditions at both interfaces, the fluid-structure $\left(\Gamma_{q}\right)$ and the soil-structure $\left(\Gamma_{o}\right)$ interfaces. The radiated wave field is computed once the displacement and traction solutions at $\Gamma_{o}$ are known.

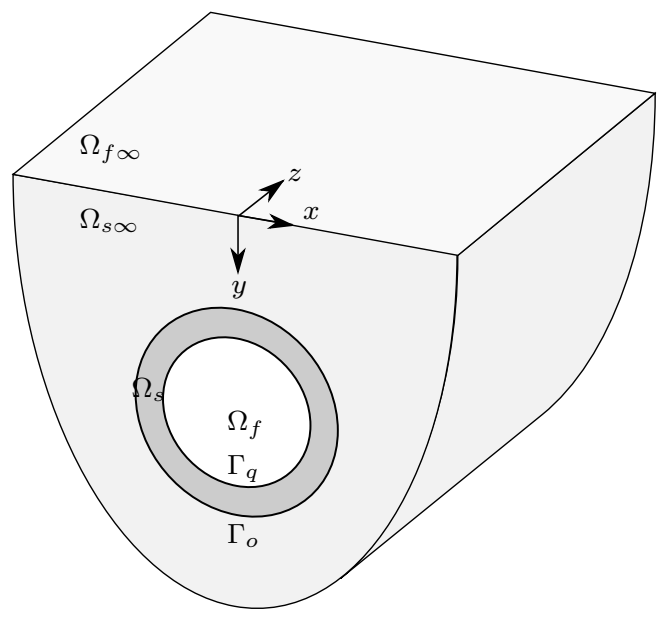

Figure 1: Problem definition (figure not scaled). Solid $\left(\Omega_{s}\right)$ and fluid $\left(\Omega_{f}\right)$ enclosures, soil domain $\left(\Omega_{s} \infty\right)$, air above the ground surface $\left(\Omega_{f \infty}\right)$ and the fluid-structure $\left(\Gamma_{q}\right)$ and the soil-structure $\left(\Gamma_{o}\right)$ interfaces.

The 2.5D formulation computes the $3 \mathrm{D}$ solution in the frequency domain, assuming that the problem is invariant in the longitudinal direction $z$, as the superposition of $2 \mathrm{D}$ problems with a different longitudinal wavenumber $\left(k_{z}\right)$ in the $z$ direction. An inverse Fourier transform is used to compute the $3 \mathrm{D}$ solution at a 
point $\mathbf{x}(x, y, z)$ :

$$
a(\mathbf{x}, \omega)=\int_{-\infty}^{+\infty} \widehat{a}\left(\widehat{\mathbf{x}}, k_{z}, \omega\right) e^{-i k_{z} z} d k_{z}
$$

where $a(\mathbf{x}, \omega)$ is the unknown variable in the frequency domain (e.g. displacement, traction or pressure), $\widehat{a}\left(\widehat{\mathbf{x}}, k_{z}, \omega\right)$ is its representation in the wavenumber-frequency domain at $\widehat{\mathbf{x}}=\mathbf{x}(x, y, 0), \omega$ is the corresponding angular frequency, and $i=\sqrt{-1}$.

The Equation (1) converges and can be approximated by a discrete Fourier transform over $k_{z}$. The wavenumber sampling $\Delta k_{z}$ defines the maximum distance $z$ where the solution is accurately computed as $\max (z)=2 \pi / \Delta k_{z} / 2$. In this work, the wavenumber sampling used in this work is uniform spaced. Moreover, the problem solution is obtained at equally spaced points with $\Delta z=2 \pi / \max \left(k_{z}\right)$. The computational effort of the spatial Fourier transform depends on these parameters.

Next, the numerical methodology is discussed. First, the BEM formulation and its fundamental solution are presented. Then, the FEM formulation for elastodynamic and fluid-acoustics are briefly described. Finally, the coupled BEM-FEM model is obtained.

\subsection{Boundary element formulation}

The boundary element formulation we present considers an arbitrary boundary inside a solid half-space. This solid half-space is bounded by a fluid medium. The solid and the fluid half-spaces are used to represent the soil domain $\left(\Omega_{s \infty}\right)$ and the air above the ground surface $\left(\Omega_{f \infty}\right)$, respectively. The arbitrary enclosure accounts for the soil-structure interface $\left(\Gamma_{o}\right)$.

The integral representation of displacement in the frequency domain for a point $\mathbf{x}^{i}$ located at the arbitrary boundary $\Gamma_{o}$ can be written as [22]:

$$
c_{l k}^{i} u_{k}^{i}\left(\mathbf{x}^{i}, \omega\right)=\int_{\Gamma_{o}} u_{l k}^{*}\left(\mathbf{x}, \omega ; \mathbf{x}^{i}\right) t_{k}^{i}(\mathbf{x}, \omega) d \Gamma-\int_{\Gamma_{o}} t_{l k}^{*}\left(\mathbf{x}, \omega ; \mathbf{x}^{i}\right) u_{k}^{i}(\mathbf{x}, \omega) d \Gamma
$$

where $u_{k}^{i}$ and $t_{k}^{i}$ are the component $k$ of displacements and tractions at the interface $\Gamma_{o}$, respectively. $u_{l k}^{*}$ and $t_{l k}^{*}$ are the fluid-solid domain fundamental solution for displacements and tractions, respectively, at point $\mathbf{x}$ due to a point load at $\mathbf{x}^{i}$ acting in the $l$ direction at the solid [21]. The integral-free term $c_{l k}^{i}$ depends only on the boundary geometry at point $\mathbf{x}^{i}[22]$.

Assuming that the boundary $\Gamma_{o}$ is invariant in the longitudinal direction $z$, Equation (2) is expressed in terms of integrals over the cross section of the boundary $\Gamma_{o}$, denoted as $\Sigma_{o}$ :

$$
c_{l k}^{i} u_{k}^{i}\left(\mathbf{x}^{i}, \omega\right)=\int_{\Sigma_{o}} \int_{-\infty}^{+\infty} u_{l k}^{*}\left(\mathbf{x}, \omega ; \mathbf{x}^{i}\right) t_{k}^{i}(\mathbf{x}, \omega) d \Sigma d z-\int_{\Sigma_{o}} \int_{-\infty}^{+\infty} t_{l k}^{*}\left(\mathbf{x}, \omega ; \mathbf{x}^{i}\right) u_{k}^{i}(\mathbf{x}, \omega) d \Sigma d z
$$

Equation (3) is then transformed to the wavenumber domain as (Equation 1): 


$$
c_{l k}^{i} \widehat{u}_{k}^{i}\left(\widehat{\mathbf{x}}^{i}, k_{z}, \omega\right)=\int_{\Sigma_{o}} \widehat{u}_{l k}^{*}\left(\widehat{\mathbf{x}}, k_{z}, \omega ; \widehat{\mathbf{x}}^{i}\right) \widehat{t}_{k}^{i}\left(\widehat{\mathbf{x}}, k_{z}, \omega\right) d \Sigma-\int_{\Sigma_{o}} \widehat{t}_{l k}^{*}\left(\widehat{\mathbf{x}}, k_{z}, \omega ; \widehat{\mathbf{x}}^{i}\right) \widehat{u}_{k}^{i}\left(\widehat{\mathbf{x}}, k_{z}, \omega\right) d \Sigma
$$

The boundary cross section $\Sigma_{o}$ is discretised into elements and nodes, leading to a boundary approximation of the displacement and traction using the interpolation shape function $N$. Then, Equation (4) is written as:

$$
c_{l k}^{i} \widehat{u}_{k}^{i}=\sum_{j=1}^{Q}\left[\left\{\int_{\Sigma_{j}} \widehat{u}_{l k}^{*} N^{j} d \Sigma\right\} \widehat{t}_{k}^{j}-\left\{\int_{\Sigma_{j}} \widehat{t}_{l k}^{*} N^{j} d \Sigma\right\} \widehat{u}_{k}^{j}\right]=\sum_{j=1}^{Q}\left[\widehat{G}_{l k}^{i j} \widehat{t}_{k}^{j}-\widehat{H}_{l k}^{i j} \widehat{u}_{k}^{j}\right]
$$

where $Q$ is the number of nodes at $\Sigma_{o}$, and $\Sigma_{j}$ stands for the elements which contain the node $j$.

The system of equations for all the boundary nodes in the wavenumber-frequency domain becomes:

$$
\widehat{\mathbf{H}}\left(k_{z}, \omega\right) \widehat{\mathbf{u}}_{o}\left(\widehat{\mathbf{x}}, k_{z}, \omega\right)=\widehat{\mathbf{G}}\left(k_{z}, \omega\right) \widehat{\mathbf{t}}_{o}\left(\widehat{\mathbf{x}}, k_{z}, \omega\right)
$$

where $\widehat{\mathbf{G}}$ and $\widehat{\mathbf{H}}$ are the fully nonsymmetrical boundary element system matrices, and $\widehat{\mathbf{u}}_{o}$ and $\widehat{\mathbf{t}}_{o}$ are displacements and tractions at the interface $\Gamma_{o}$, repectively.

In this work, constant boundary elements are used. The boundary integrals are computed using an eight point Gauss-Legendre quadrature whenever the collocation point does not belong to the integration element. Closed forms are used to solve singular integrals when the collocation point $\widehat{\mathbf{x}}^{i}$ belongs to the integration element. The exact expressions for the singular integrals given in References $\underline{[23,24]}$ are used. The analytical solution for the steady state response of a formation composed by a homogeneous solid half-space bounded by a fluid medium subjected to a spatially harmonic load can be found in Reference [21]. This solution relates displacement at point $\mathbf{x}(x, y, z)$ due to a dynamic source placed at a different point $\mathbf{x}^{i}\left(x^{i}, y^{i}, z^{i}\right)$ in the fluid-solid formation (Figure 2).

The boundary element collocation method described by Equation (5) requires evaluation of the fundamental solution at each Gaussian integration point for sources placed at every nodal collocation point. This procedure could be highly expensive, depending on the size of the soil-structure interface discretisation. In this section, the Green's function presented in Reference $\underline{[21]}$ is modified in a feasible form to carry out the BEM collocation methodology.

The Green's function $(\mathcal{G})$ given by a spatially sinusoidal harmonic load in the $z$ direction in the frequency domain is expressed as the superposition of $2 \mathrm{D}$ problems with the longitudinal wavenumber $k_{z}$ varying in the $z$ direction:

$$
\mathcal{G}\left(\mathbf{x}, \omega ; \mathbf{x}^{i}\right)=\int_{-\infty}^{+\infty} \widehat{\mathcal{G}}\left(\widehat{\mathbf{x}}, k_{z}, \omega ; \widehat{\mathbf{x}}^{i}\right) e^{-i k_{z}\left(z-z^{i}\right)} d k_{z}
$$

where $\widehat{\mathcal{G}}$ represents the Green's function in the wavenumber-frequency domain.

The Green's function for the fluid-solid formation is derived by imposing compatibility and equilibrium of normal displacements and stresses, and assuming null shear stresses, at the interface between the solid 


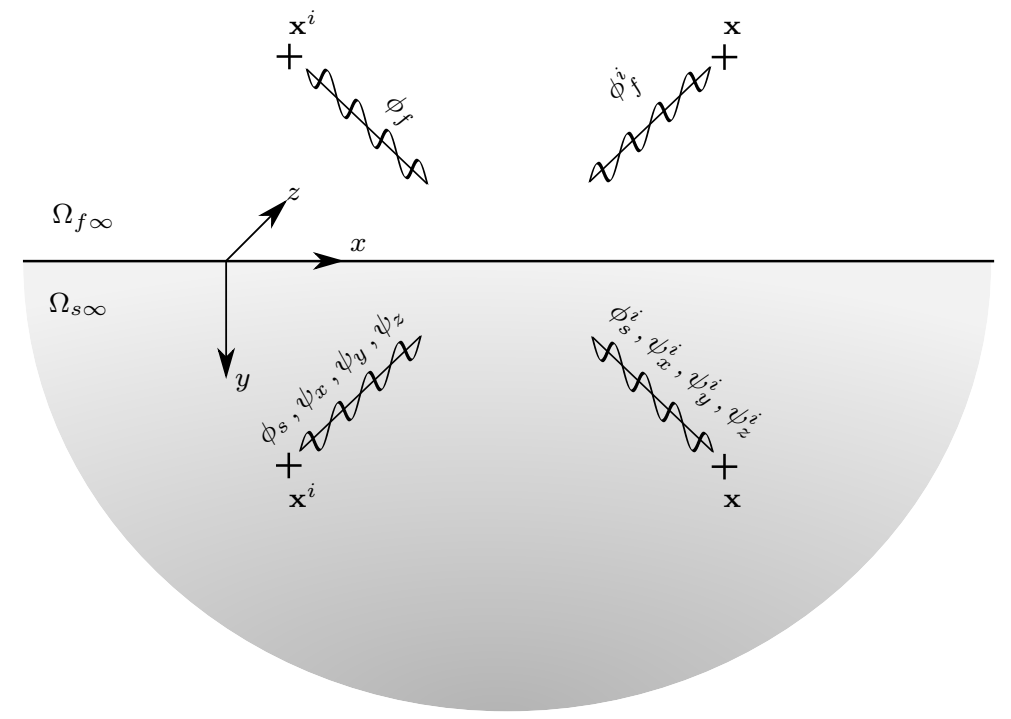

Figure 2: Solid formation bounded by a flat fluid medium (figure not scaled). Soil domain $\left(\Omega_{s \infty}\right)$, air above the ground surface $\left(\Omega_{f \infty}\right)$, source point $\left(\mathbf{x}^{i}\left(x^{i}, y^{i}, z^{i}\right)\right)$, observation point $(\mathbf{x}(x, y, z))$, solid compressional $\left(\phi_{s}, \phi_{s}^{i}\right)$ and rotational $\left(\psi_{x}, \psi_{y}, \psi_{z}, \psi_{x}^{i}\right.$, $\left.\psi_{y}^{i}, \psi_{z}^{i}\right)$ potentials and fluid dilatational potential $\left(\phi_{f}, \phi_{f}^{i}\right)$.

and the fluid medium. The function can be expressed as the superposition of: (i) source terms equal to those in the full-space $\left(\widehat{\mathcal{G}}^{\text {full }}\right)$, and (ii) surface terms $\left(\widehat{\mathcal{G}}^{\text {surf }}\right)$ needed to represent the indirect wave field scattered by the fluid-solid interface [21]:

$$
\widehat{\mathcal{G}}\left(\widehat{\mathbf{x}}, k_{z}, \omega ; \widehat{\mathbf{x}}^{i}\right)=\widehat{\mathcal{G}}^{\text {full }}\left(\widehat{\mathbf{x}}, k_{z}, \omega ; \widehat{\mathbf{x}}^{i}\right)+\widehat{\mathcal{G}}^{\text {surf }}\left(\widehat{\mathbf{x}}, k_{z}, \omega ; \widehat{\mathbf{x}}^{i}\right)
$$

The expressions for the Green's function for the full-space ( $\widehat{\mathcal{G}}^{\text {full }}$ ) are defined explicitly in Reference [25]. The source terms are obtained from compressional $\left(\phi_{s}\right)$ and rotational $\left(\psi_{x}, \psi_{y}, \psi_{z}\right)$ potentials for a solid full-space. The fluid dilatational potential $\left(\phi_{f}\right)$ for a harmonic pressure load acting on an unbounded fluid medium defines the Green's function for the direct pressure field. The meaning of these potentials is well established in Reference [21].

The surface terms can be expressed in the same way as those of the source terms using the potentials $\phi_{s}^{i}, \psi_{x}^{i}, \psi_{y}^{i}, \psi_{z}^{i}$, and $\phi_{f}^{i}$. Each potential amplitude can be computed imposing the four stated boundary conditions at the fluid-solid interface $(y=0)$ :

$$
\left\{\begin{array}{l}
\sigma_{y x}^{s}=\sigma_{y x}^{f}=0 \\
\sigma_{y z}^{s}=\sigma_{y z}^{f}=0 \\
\sigma_{y y}^{s}=\sigma_{y y}^{f} \\
u_{y}^{s}=u_{y}^{f}
\end{array}\right.
$$

where superscripts $s$ and $f$ relate to variables belonging to the solid and fluid subdomains, respectively. 
The surface terms are given by a spatial Fourier transform in the $x$ direction for the wavenumber $k_{x}$, which can be approximated by a finite sum of terms:

$$
\widehat{\mathcal{G}}^{\operatorname{surf}}\left(\widehat{\mathbf{x}}, k_{z}, \omega ; \widehat{\mathbf{x}}^{i}\right)=\int_{-\infty}^{+\infty} \widetilde{\mathcal{G}}^{\text {surf }}\left(k_{x}, y, k_{z}, \omega ; y^{i}\right) e^{-i k_{x}\left(x-x^{i}\right)} d k_{x}
$$

Following the approach given in Equation (10), the expressions for solid displacements due to a load acting in the $x$ direction are defined as $\underline{[21]}$ :

$$
\left\{\begin{array}{l}
\widetilde{\mathcal{G}}_{x x}^{\text {surf }}=E_{a}\left[A^{x} \frac{-i k_{x}^{2}}{\nu_{x}^{s}} E_{b}+\left(-i \gamma_{x} C^{x}-\frac{i k_{z}}{\gamma_{x}} B^{x}\right) E_{c}\right] \\
\widetilde{\mathcal{G}}_{y x}^{\text {surf }}=E_{a}\left(-i k_{x} A^{x} E_{b}+i k_{x} C^{x} E_{c}\right) \\
\widetilde{\mathcal{G}}_{z x}^{\text {surf }}=E_{a}\left(\frac{-i k_{z} k_{x}}{\nu_{x}^{s}} A^{x} E_{b}+\frac{i k_{z} k_{x}}{\gamma_{x}} B^{x} E_{c}\right)
\end{array}\right.
$$

where $E_{a}=1 / 2 \rho_{s} \omega^{2}, E_{b}=e^{-i \nu_{x}^{s} y}, E_{c}=e^{-i \gamma_{x} y}, \nu_{x}^{s}=\sqrt{k_{p}^{2}-k_{z}^{2}-k_{x}^{2}}$ with $\left(\operatorname{Im}\left(\nu_{x}^{s}\right) \leq 0\right)$, and $\gamma_{x}=$ $\sqrt{k_{s}^{2}-k_{z}^{2}-k_{x}^{2}}$ with $\left(\operatorname{Im}\left(\gamma_{x}\right) \leq 0\right)$. The wavenumbers related to $\mathrm{P}$ and $\mathrm{S}$ waves are $k_{p}=\omega / c_{p}$ and $k_{s}=\omega / c_{s}$, respectively, $\rho_{s}$ is the solid density, and $c_{p}$ and $c_{s}$ are the propagation velocity of $\mathrm{P}$ and $\mathrm{S}$ waves in the solid, respectively. $A^{x}, B^{x}$, and $C^{x}$ are unknown coefficients to be determined from the conditions defined by Equation (9). The imposition of the four boundary conditions leads to the following two systems of four equations $[21]$ :

$$
\left[\begin{array}{cccc}
-2 k_{x}^{2} & -k_{z}^{2} & k_{x}^{2}-\gamma_{x}^{2} & 0 \\
-2 & 1 & 1 & 0 \\
\frac{-k_{s}^{2}-2 \nu_{z x}^{2}}{\nu_{x}^{s}} & 0 & 2 \gamma_{x} & \frac{-i 2 \rho_{s} \omega^{2}}{\nu_{x}^{f} \mu_{s}} \\
-i & 0 & i & \frac{-2 \rho_{s} \omega^{2}}{k_{f}^{2} \lambda_{f}}
\end{array}\right]\left[\begin{array}{c}
\overline{\mathbf{A}}^{x} \\
\overline{\mathbf{B}}^{x} \\
\overline{\mathbf{C}}^{x} \\
\overline{\mathbf{D}}^{x}
\end{array}\right]=\left[\begin{array}{c}
-2 k_{x}^{2} \\
-2 \\
\left(\frac{k_{s}^{2}+2 \nu_{z x}^{2}}{\nu_{x}^{s}}\right) \\
-i
\end{array}\right.
$$

where $\mu_{s}$ is the solid second Lamé constant, $\lambda_{f}$ is the fluid Lamé constant, $\nu_{x}^{f}=\sqrt{k_{f}^{2}-k_{z}^{2}-k_{x}^{2}}$ with $\left(\operatorname{Im}\left(\nu_{x}^{f}\right) \leq 0\right), \nu_{z x}=\sqrt{-k_{z}^{2}-k_{x}^{2}}$, and the wavenumber related to the fluid pressure wave is $k_{f}=\omega / c_{f}$, with $c_{f}$ being the fluid compressional wave velocity.

After the solution vectors $\overline{\mathbf{A}}^{x}, \overline{\mathbf{B}}^{x}, \overline{\mathbf{C}}^{x}$ and $\overline{\mathbf{D}}^{x}$ are computed, the amplitudes of the potentials are given by the following expressions:

$$
\left\{\begin{array}{l}
A^{x}=E_{b}^{i} \overline{\mathbf{A}}^{x}(1)+E_{c}^{i} \overline{\mathbf{A}}^{x}(2) \\
B^{x}=E_{b}^{i} \overline{\mathbf{B}}^{x}(1)+E_{c}^{i} \overline{\mathbf{B}}^{x}(2) \\
C^{x}=E_{b}^{i} \overline{\mathbf{C}}^{x}(1)+E_{c}^{i} \overline{\mathbf{C}}^{x}(2)
\end{array}\right.
$$

with $E_{b}^{i}=e^{-i \nu_{x}^{s} y^{i}}$ and $E_{c}^{i}=e^{-i \gamma_{x} y^{i}}$. The fluid pressure is computed from $\overline{\mathbf{D}}^{x} \underline{[21]}$. 
In the same way, the fundamental solution for a load acting in the $y$ direction is expressed through the following equations:

$$
\left\{\begin{array}{l}
\widetilde{\mathcal{G}}_{x y}^{\text {surf }}=E_{a}\left[-i A^{y} k_{x} E_{b}+i B^{y} k_{x} E_{c}\right] \\
\widetilde{\mathcal{G}}_{y y}^{\text {surf }}=E_{a}\left[-i \nu_{x}^{s} A^{y} E_{b}+\left(\frac{-i k_{x}^{2}}{\gamma_{x}} B^{y}+\frac{-i k_{z}^{2}}{\gamma_{x}} C^{y}\right) E_{c}\right] \\
\widetilde{\mathcal{G}}_{z y}^{\text {surf }}=E_{a}\left[-i A^{y} k_{z} E_{b}+i C^{y} k_{z} E_{c}\right]
\end{array}\right.
$$

where the amplitudes of potentials are defined by:

$$
\left[\begin{array}{cccc}
-2 \nu_{x}^{s} & \frac{-k_{x}^{2}}{\gamma_{x}}+\gamma_{x} & \frac{-k_{z}^{2}}{\gamma_{x}} & 0 \\
-2 \nu_{x}^{s} & \frac{-k_{x}^{2}}{\gamma_{x}} & \frac{-k_{z}^{2}}{\gamma_{x}}+\gamma_{x} & 0 \\
-k_{s}^{2}-2 \nu_{z x}^{2} & -2 k_{x}^{2} & -2 k_{z}^{2} & \frac{-i 2 \rho_{s} \omega^{2}}{\nu_{x}^{f} \mu_{s}} \\
-i \nu_{x}^{s} & \frac{-i k_{x}^{2}}{\gamma_{x}} & \frac{-i k_{z}^{2}}{\gamma_{x}} & \frac{-i 2 \rho_{s} \omega^{2}}{k_{f}^{2} \lambda_{f}}
\end{array}\right]\left[\begin{array}{c}
\overline{\mathbf{A}}^{y} \\
\overline{\mathbf{B}}^{y} \\
\overline{\mathbf{C}}^{y} \\
\overline{\mathbf{D}}^{y}
\end{array}\right]=\left[\begin{array}{cc}
2 \nu_{x}^{s} & -\left(\frac{\nu_{z x}^{2}}{\gamma_{x}}+\gamma_{x}\right) \\
2 \nu_{x}^{s} & -\left(\frac{\nu_{z x}^{2}}{\gamma_{x}}+\gamma_{x}\right) \\
2 \nu_{z x}^{2} \\
\left(-k_{s}^{2}-2 \nu_{z x}^{2}\right) & i \nu_{x}^{s} \\
\left.i \frac{i k_{x}^{2}+i k_{z}^{2}}{\gamma_{x}}\right)
\end{array}\right]
$$

and:

$$
\left\{\begin{array}{l}
A^{y}=E_{b}^{i} \overline{\mathbf{A}}^{y}(1)+E_{c}^{i} \overline{\mathbf{A}}^{y}(2) \\
B^{y}=E_{b}^{i} \overline{\mathbf{B}}^{y}(1)+E_{c}^{i} \overline{\mathbf{B}}^{y}(2) \\
C^{y}=E_{b}^{i} \overline{\mathbf{C}}^{y}(1)+E_{c}^{i} \overline{\mathbf{C}}^{y}(2)
\end{array}\right.
$$

Furthermore, solid displacements for a load acting in the $z$ direction are obtained as follows:

$$
\left\{\begin{array}{l}
\widetilde{\mathcal{G}}_{x z}^{\text {surf }}=E_{a}\left[\frac{-i k_{z} k_{x}}{\nu_{x}^{s}} A^{z} E_{b}+\frac{i k_{z} k_{x}}{\gamma_{x}} C^{z} E_{c}\right] \\
\widetilde{\mathcal{G}}_{y z}^{\text {surf }}=E_{a}\left[-i k_{z} A^{z} E_{b}+i B^{z} k_{z} E_{c}\right] \\
\widetilde{\mathcal{G}}_{z z}^{\text {surf }}=E_{a}\left[\frac{-i k_{z}^{2}}{\nu_{x}^{s}} A^{z} E_{b}+\left(\frac{-i k_{x}^{2}}{\gamma_{x}} C^{z}-i \gamma_{x} B^{z}\right) E_{c}\right]
\end{array}\right.
$$

The amplitudes of the potentials are provided by the following system of equations:

$$
\left[\begin{array}{cccc}
-2 & 1 & 1 & 0 \\
-2 k_{z}^{2} & k_{z}^{2}-\gamma_{x}^{2} & -k_{x}^{2} & 0 \\
-\frac{k_{s}^{2}+2 \nu_{z x}^{2}}{\nu_{x}^{s}} & 2 \gamma_{x} & 0 & \frac{-i 2 \rho_{s} \omega^{2}}{\nu_{x}^{f} k_{z} \mu_{s}} \\
-i k_{z} & i k_{z} & 0 & \frac{-2 \rho_{s} \omega^{2}}{k_{f}^{2} \lambda_{f}}
\end{array}\right]\left[\begin{array}{c}
\overline{\mathbf{A}}^{z} \\
\overline{\mathbf{B}}^{z} \\
\overline{\mathbf{C}}^{z} \\
\overline{\mathbf{D}}^{z}
\end{array}\right]=\left[\begin{array}{cc}
-2 & 2 \\
-2 k_{z}^{2} & \left(k_{z}^{2}-\gamma_{x}-k_{x}^{2}\right) \\
\left(\frac{k_{s}^{2}+2 \nu_{z x}^{2}}{\nu_{x}^{s}}\right) & -2 \gamma_{x} \\
-i k_{z} & i k_{z}
\end{array}\right]
$$

thus,

$$
\left\{\begin{array}{l}
A^{z}=E_{b}^{i} \overline{\mathbf{A}}^{z}(1)+E_{c}^{i} \overline{\mathbf{A}}^{z}(2) \\
B^{z}=E_{b}^{i} \overline{\mathbf{B}}^{z}(1)+E_{c}^{i} \overline{\mathbf{B}}^{z}(2) \\
C^{z}=E_{b}^{i} \overline{\mathbf{C}}^{z}(1)+E_{c}^{i} \overline{\mathbf{C}}^{z}(2)
\end{array}\right.
$$


Finally, the well-known equations relating strains and displacements, and strain and stresses are used to calculate expressions for stress in the solid formation.

The computational effort to evaluate the BEM Equation (5) can be reduced taking into account that the surface terms $\widehat{\mathcal{G}}^{\text {surf }}$ (Equation (10)) are given by a Fourier transform in the $x$ direction. In this way, the surface terms $\widetilde{\mathcal{G}}^{\text {surf }}$ written in their current form do not depend on the horizontal distance between the source and the observation point. Moreover, potential amplitudes only depend on the source location coordinate $y^{i}$ and they are computed independently of the receiver position. The surface terms defined by Equations (11), (14) and (17) are then only evaluated for a single set of sources and receivers (Figure 3).This methodology considerably reduces the computational time. The computation of source terms $\widehat{\mathcal{G}}^{\text {full }}$ takes much less time than the computation of the surface terms.

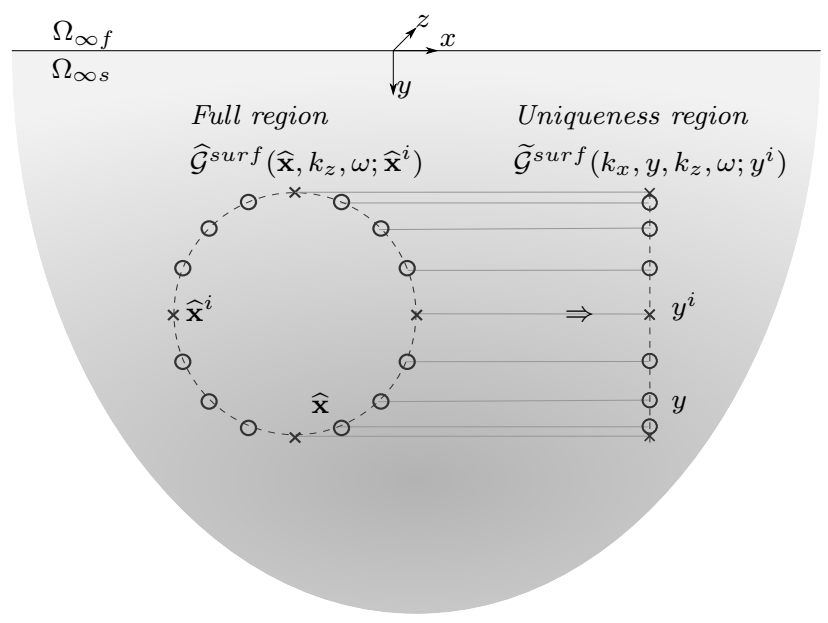

Figure 3: Collocation nodes (crosses) and Gauss integration points (circles) for the full region and the uniqueness region (figure not scaled). Soil domain $\left(\Omega_{s \infty}\right)$, air above the ground surface $\left(\Omega_{f \infty}\right)$, surface terms $\left(\widehat{\mathcal{G}}^{\text {surf }}\right.$ and $\left.\widetilde{\mathcal{G}}^{\text {surf }}\right)$.

The proposed model only accounts for the radiation soil damping according to the Sommerfield condition. The internal soil damping could be considered through a hysteretic damping model by complex wave propagation velocities [22]. The solution for layered soils would be obtained by the superposition of the surface terms contribution defined for each layer interface. These terms could be expressed as the sum of dilatational and rotational potentials of a solid layer similarly to those described in this section [26].

\subsection{Finite element formulation for fluid-acoustics}

The FEM wavenumber-frequency domain formulation that was previously presented by the authors in Reference $[20]$ is very briefly summarized in this section. The $2.5 \mathrm{D}$ governing equation in fluid-acoustics is derived from the 3D formulation, assuming that the fluid subdomain is invariant in the longitudinal direction [20]. Wave propagation within an inviscid fluid is expressed by the homogeneous Helmholtz equation [27], 
and the energy lost at the fluid boundary enclosure can be represented by a resistivity term [28]. The finite element equation is then derived from a virtual differential of pressure $\delta p$ acting on the subdomain $\Omega_{f}$ :

$$
\int_{\Omega_{f}} \delta p(\mathbf{x}, \omega) \nabla^{2} p(\mathbf{x}, \omega) d \Omega+k^{2} \int_{\Omega_{f}} \delta p(\mathbf{x}, \omega) p(\mathbf{x}, \omega) d \Omega-i k \beta \int_{\Omega_{f}} \delta p(\mathbf{x}, \omega) p(\mathbf{x}, \omega) d \Omega=0
$$

where $k=\omega / c_{f}$ is the wavenumber, $c_{f}$ is the fluid compressional wave velocity, $p$ is the fluid pressure, and $\beta$ is the fluid absorption coefficient.

The FEM equation is addressed on the assumption that the fluid subdomain $\left(\Omega_{f}\right)$ is invariant along the $z$ coordinate. The cross section of $\Omega_{f}$ is discretised into elements, and the pressures are approximated within every element by interpolation shape functions. The fluid momentum equation yields a relationship between pressure and particle displacement, involving spatial derivatives that are solved by a Fourier transform along the longitudinal coordinate $z$. Then, the FEM equation is approached by [20]:

$$
\left[-\left(k^{2}-i k \beta\right) \mathbf{D}+\mathbf{F}^{0}-i k_{z} \mathbf{F}^{1}-k_{z}^{2} \mathbf{F}^{2}\right] \widehat{p}\left(\widehat{\mathbf{x}}, k_{z}, \omega\right)=\omega^{2} \rho_{f} \mathbf{R} \widehat{\mathbf{u}}_{q}\left(\widehat{\mathbf{x}}, k_{z}, \omega\right)
$$

where $\widehat{\mathbf{u}}_{q}$ is the displacement vector at the fluid-solid interface $\Gamma_{q}, \rho_{f}$ is the fluid density, and the matrices $\mathbf{D}, \mathbf{F}^{0}, \mathbf{F}^{1}, \mathbf{F}^{2}$, and $\mathbf{R}$ are properly defined in Reference $[20]$. The matrix $\omega^{2} \rho_{f} \mathbf{R}$ is the coupling fluid-mass matrix, and relates boundary displacements at the interface $\Gamma_{q}$ to pressures in the subdomain $\Omega_{f}$.

Finally, Equation (21) is rewritten considering an equivalent dynamic fluid matrix $(\widehat{\mathbf{F}})$ as:

$$
\widehat{\mathbf{F}}\left(k_{z}, \omega\right) \widehat{p}\left(\widehat{\mathbf{x}}, k_{z}, \omega\right)=\omega^{2} \rho_{f} \mathbf{R} \widehat{\mathbf{u}}_{q}\left(\widehat{\mathbf{x}}, k_{z}, \omega\right)
$$

where the matrix $\widehat{\mathbf{F}}$ is computed for each wavenumber and frequency.

\subsection{Finite element formulation for elastodynamics}

The solid FEM frequency domain formulation is based on the virtual work principle [29]:

$$
\begin{aligned}
& -\omega^{2} \int_{\Omega_{s}} \delta \mathbf{u}(\mathbf{x}, \omega) \rho_{s} \mathbf{u}(\omega, \mathbf{x}) d \Omega+\int_{\Omega_{s}} \delta \varepsilon(\mathbf{x}, \omega) \boldsymbol{\sigma}(\mathbf{x}, \omega) d \Omega \\
& =\int_{\Omega_{s}} \delta \mathbf{u}(\mathbf{x}, \omega) \rho_{s} \mathbf{b}(\mathbf{x}, \omega) d \Omega+\int_{\Gamma_{o}} \delta \mathbf{u}(\mathbf{x}, \omega) \mathbf{f}(\mathbf{x}, \omega) d \Gamma
\end{aligned}
$$

where $\mathbf{u}$ is the displacement vector, $\boldsymbol{\varepsilon}$ and $\boldsymbol{\sigma}$ are respectively the strain and stress tensors, $\rho_{s} \mathbf{b}$ is the body force and $\mathbf{f}$ is the nodal force. A variable preceded by $\delta$ denotes again a virtual change of this magnitude.

Once the displacements are approximated within every element by interpolation shape functions, the stress and strain vectors are derived from displacements through the constitutive law of material, assuming linear behaviour and a homogeneous medium. The spatial derivatives involved in the strain-displacement relation are solved by means of a Fourier transform. Afterwards these procedures, Equation (23) is written as [13]: 


$$
\left[-\omega^{2} \mathbf{M}+\mathbf{K}^{0}-i k_{z} \mathbf{K}^{1}-k_{z}^{2} \mathbf{K}^{2}+i k_{z}^{3} \mathbf{K}^{3}+k_{z}^{4} \mathbf{K}^{4}\right] \widehat{\mathbf{u}}\left(\widehat{\mathbf{x}}, k_{z}, \omega\right)=\widehat{\mathbf{f}}\left(\widehat{\mathbf{x}}, k_{z}, \omega\right)
$$

where $\mathbf{M}$ is the mass matrix and $\mathbf{K}^{0}, \mathbf{K}^{1}, \mathbf{K}^{2}, \mathbf{K}^{3}$ and $\mathbf{K}^{4}$ are the stiffness matrices. The meanings of these matrices are well established in References [13, 29, 30].

Equation (24) is rewritten as shown below if an equivalent dynamic stiffness matrix $(\widehat{\mathbf{K}})$ is considered:

$$
\widehat{\mathbf{K}}\left(k_{z}, \omega\right) \widehat{\mathbf{u}}\left(\widehat{\mathbf{x}}, k_{z}, \omega\right)=\widehat{\mathbf{f}}\left(\widehat{\mathbf{x}}, k_{z}, \omega\right)
$$

\subsection{BEM-FEM coupling procedure}

Equations (6), (22) and (25) are coupled under imposed force equilibrium and displacement compatibility at the soil-structure interface $\Gamma_{o}$. Also, the equilibrium and compatibility of normal pressure and displacement, and null shear stresses at the interface $\Gamma_{q}$ must be ensured. These equations are assembled into a single comprehensive system, together with the equilibrium and compatibility conditions.

Firstly, the coupling of the BEM and the FEM solid subdomains leads to an assembled system of equations that represents the soil-structure interaction problem. Equilibrium of forces at the interface $\Gamma_{o}$ is fulfilled integrating nodal tractions according to the element shape function $\mathbf{N}$ :

$$
\widehat{\mathbf{f}}_{o}=\int_{\Gamma_{o}} \mathbf{N}^{T} \widehat{\mathbf{t}}_{o} \mathbf{N} d \Gamma=\widehat{\mathbf{T t}}_{o}
$$

Substituting Equation (6) into Equation (26) yields:

$$
\widehat{\mathbf{f}}_{o}=\mathbf{T} \widehat{\mathbf{G}}^{-1} \widehat{\mathbf{H}} \widehat{\mathbf{u}}_{o}
$$

Equation (27) defines a boundary element stiffness matrix $\widehat{\mathbf{K}}_{o}$ that relates nodal displacements and forces [13]:

$$
\widehat{\mathbf{K}}_{o}\left(k_{z}, \omega\right) \widehat{\mathbf{u}}_{o}\left(\widehat{\mathbf{x}}, k_{z}, \omega\right)=\widehat{\mathbf{f}}_{o}\left(\widehat{\mathbf{x}}, k_{z}, \omega\right)
$$

Equations (25) and (28) are assembled into a global system of equations by means of the equilibrium and compatibility conditions at the interface $\Gamma_{o}$ :

$$
\widehat{\mathbf{D}}\left(k_{z}, \omega\right) \widehat{\mathbf{u}}\left(\widehat{\mathbf{x}}, k_{z}, \omega\right)=\widehat{\mathbf{f}}\left(\widehat{\mathbf{x}}, k_{z}, \omega\right)
$$

where $\widehat{\mathbf{D}}$ is the coupled BEM-FEM matrix which describes the soil-structure interaction problem.

On the other hand, the load vector $\widehat{\mathbf{f}}_{q}$ at the fluid-solid interface is obtained by pressure integration over $\Gamma_{q} \underline{[20]:}$ 


$$
\widehat{\mathbf{f}}_{q}=\int_{\Gamma_{q}} \mathbf{N}^{T} \mathbf{n}_{q} \mathbf{N} \widehat{p}_{q} d \Gamma=\mathbf{R}^{T} \widehat{p}_{q}
$$

where $\widehat{p}_{q}$ is the fluid pressure at the interface $\Gamma_{q}$, and $\mathbf{n}_{q}$ is the outward normal to $\Gamma_{q}$.

The coupling of Equations (22) and (29) is carried out with the imposition of equilibrium and compatibility conditions of normal pressure and displacement at the interface $\Gamma_{q}$, and null shear stresses. Equations (22) and (29) are split in two parts according to the boundary subdomain definition (Figure 1). Both systems of equations are assembled into an overall system:

$$
\left[\begin{array}{cccc}
\widehat{\mathbf{D}}_{s s} & \widehat{\mathbf{D}}_{s q} & \mathbf{0} & \mathbf{0} \\
\widehat{\mathbf{D}}_{q s} & \widehat{\mathbf{D}}_{q q} & -\mathbf{R}^{T} & \mathbf{0} \\
\mathbf{0} & -\omega^{2} \rho \mathbf{R} & \widehat{\mathbf{F}}_{q q} & \widehat{\mathbf{F}}_{q f} \\
\mathbf{0} & \mathbf{0} & \widehat{\mathbf{F}}_{f q} & \widehat{\mathbf{F}}_{f f}
\end{array}\right]\left[\begin{array}{c}
\widehat{\mathbf{u}}_{s} \\
\widehat{\mathbf{u}}_{q} \\
\widehat{p}_{q} \\
\widehat{p}_{f}
\end{array}\right]=\left[\begin{array}{c}
\widehat{\mathbf{f}}_{s} \\
\mathbf{0} \\
\mathbf{0} \\
\mathbf{0}
\end{array}\right]
$$

where subscript $q$ indicates degrees of freedom belonging to the fluid-solid interface $\Gamma_{q}, s$ stands for the other solid degrees of freedom, and $f$ represents the rest of fluid degrees of freedom. Equation (31) is solved using a direct solver for each frequency-wavenumber step to compute the coupled fluid-solid response.

\subsection{Radiated wavefield}

After the interaction problem has been solved, the radiated wave field is computed by means of the Somigliana identity. Soil displacements $\widehat{\mathbf{u}}_{r}$ at receiver locations $\Omega_{s \infty}$ are computed as:

$$
\widehat{\mathbf{u}}_{r}\left(\widehat{\mathbf{x}}, k_{z}, \omega\right)=\widehat{\mathbf{G}}_{r}^{s}\left(k_{z}, \omega\right) \widehat{\mathbf{t}}_{o}\left(\widehat{\mathbf{x}}, k_{z}, \omega\right)-\widehat{\mathbf{H}}_{r}^{s}\left(k_{z}, \omega\right) \widehat{\mathbf{u}}_{o}\left(\widehat{\mathbf{x}}, k_{z}, \omega\right)
$$

where $\widehat{\mathbf{G}}_{r}^{s}$ and $\widehat{\mathbf{H}}_{r}^{s}$ are obtained from Equation (5) using receivers as collocation points.

Moreover, the radiated field at $\Omega_{f \infty}$ is computed using the integral representation of the pressure for a point $\mathbf{x}^{i}$ located in the fluid formation:

$$
\widehat{p}^{i}\left(\widehat{\mathbf{x}}^{i}, k_{z}, \omega\right)=\int_{\Sigma_{s}} \widehat{t}_{f k}^{*}\left(\widehat{\mathbf{x}}, k_{z}, \omega ; \widehat{\mathbf{x}}^{i}\right) \widehat{u}_{k}^{i}\left(\widehat{\mathbf{x}}, k_{z}, \omega\right) d \Sigma-\int_{\Sigma_{s}} \widehat{u}_{f k}^{*}\left(\widehat{\mathbf{x}}, k_{z}, \omega ; \widehat{\mathbf{x}}^{i}\right) \widehat{t}_{k}^{i}\left(\widehat{\mathbf{x}}, k_{z}, \omega\right) d \Sigma
$$

where $\widehat{u}_{k}^{i}\left(\widehat{\mathbf{x}}, k_{z}, \omega\right)$ and $\widehat{t}_{k}^{i}\left(\widehat{\mathbf{x}}, k_{z}, \omega\right)$ are the boundary solutions, and $\widehat{u}_{f k}^{*}\left(\widehat{\mathbf{x}}, k_{z}, \omega ; \widehat{\mathbf{x}}^{i}\right)$ and $\widehat{t}_{f k}^{*}\left(\widehat{\mathbf{x}}, k_{z}, \omega ; \widehat{\mathbf{x}}^{i}\right)$ are the fundamental solutions for displacements and tractions, respectively, at a point $\widehat{\mathbf{x}}$ in the solid due to a pressure load acting at point $\widehat{\mathbf{x}}^{i}$ in the fluid.

In this case, the fundamental solution is only defined by surface terms [21]: 


$$
\left\{\begin{array}{l}
\widetilde{\mathcal{G}}_{x f}^{\text {surf }}=E_{a}\left(-i A^{f} k_{x} E_{b}+i B^{f} k_{x} E_{c}\right) \\
\widetilde{\mathcal{G}}_{y f}^{\text {surf }}=E_{a}\left[-i \nu_{x}^{s} A^{f} E_{b}+\left(\frac{-i k_{x}^{2}}{\gamma_{x}} B^{f}+\frac{-i k_{z}^{2}}{\gamma_{x}} C^{f}\right) E_{c}\right] \\
\widetilde{\mathcal{G}}_{z f}^{\text {surf }}=E_{a}\left(-i A^{f} k_{z} E_{b}+i C^{f} k_{z} E_{c}\right)
\end{array}\right.
$$

where the amplitudes of each potential $\left(A^{f}, B^{f}, C^{f}\right.$,and $\left.D^{f}\right)$ are determined by imposing the four stated boundary conditions (Equation (9)) as [21]:

$$
\left[\begin{array}{cccc}
-2 \nu_{x}^{s} & \frac{-k_{x}^{s 2}}{\gamma_{x}}+\gamma_{x} & \frac{-k_{z}^{2}}{\gamma_{x}} & 0 \\
-2 \nu_{x}^{s} & \frac{-k_{x}^{s 2}}{\gamma_{x}} & \frac{-k_{z}^{2}}{\gamma_{x}}+\gamma_{x} & 0 \\
-k_{s}^{2}-2 \nu_{z x}^{2} & -2 k_{x}^{2} & -2 k_{z}^{2} & \frac{-i 2 \rho_{s} \omega^{2}}{\nu_{x}^{f} \mu_{s}} \\
-i \nu_{x}^{s} & \frac{-i k_{x}^{2}}{\gamma_{x}} & \frac{-i k_{z}^{2}}{\gamma_{x}} & \frac{-2 \rho_{s} \omega^{2}}{k_{f}^{2} \lambda_{f}}
\end{array}\right]\left[\begin{array}{c}
A^{f} \\
B^{f} \\
C^{f} \\
D^{f}
\end{array}\right]=\left[\begin{array}{c}
0 \\
0 \\
\frac{-i 2 \rho_{s} \omega^{2}}{\nu_{x}^{f} \mu_{s}} \\
\frac{2 \rho_{s} \omega^{2}}{k_{f}^{2} \lambda_{f}}
\end{array}\right]
$$

After solving Equation (35), each potential amplitude is computed by multiplication with the term $E_{f}^{i}=$ $e^{-i \nu_{x}^{f} y^{i}}$

Finally, pressure field $\widehat{p}_{r}$ at the fluid receivers is computed by Equation (33) as:

$$
\widehat{p}_{r}\left(\widehat{\mathbf{x}}, k_{z}, \omega\right)=\widehat{\mathbf{H}}_{r}^{f}\left(k_{z}, \omega\right) \widehat{\mathbf{u}}_{o}\left(\widehat{\mathbf{x}}, k_{z}, \omega\right)-\widehat{\mathbf{G}}_{r}^{f}\left(k_{z}, \omega\right) \widehat{\mathbf{t}}_{o}\left(\widehat{\mathbf{x}}, k_{z}, \omega\right)
$$

where $\widehat{\mathbf{G}}_{r}^{f}$ and $\widehat{\mathbf{H}}_{r}^{f}$ are obtained using fluid receivers as collocation points.

\section{Numerical verification}

The example studied in this section concerns a fluid-solid formation elicited by a spatially harmonic varying line load buried in the solid formation (Figure 4). This problem was solved by assuming a finite solid subdomain $\left(\Omega_{s}\right)$ with same properties as the unbounded solid domain $\left(\Omega_{s \infty}\right)$, which were both represented with the proposed formulation. The fluid-solid formation is excited by a harmonic point source applied in direction $y$, located at source point $S_{o}(0,2)$. Solid displacement at receiver location $R_{1}, x=2.0 \mathrm{~m}$ and $y=5.0 \mathrm{~m}$, and fluid pressure at receiver $R_{2}, x=2.0 \mathrm{~m}$ and $y=-1.0 \mathrm{~m}$, were analysed. The analytical solution can be found in Reference [21].

The fluid medium allows $c_{f}=1500 \mathrm{~m} / \mathrm{s}$ with $\rho_{f}=1000 \mathrm{~kg} / \mathrm{m}^{3}$, while the solid medium takes $c_{p}=$ $4208 \mathrm{~m} / \mathrm{s}, c_{s}=2656 \mathrm{~m} / \mathrm{s}$, and $\rho_{s}=2140 \mathrm{~kg} / \mathrm{m}^{3}$. The analysis was carried out in a frequency range of $2.5 \mathrm{~Hz}$ to $320 \mathrm{~Hz}$, with a frequency step $\Delta f=2.5 \mathrm{~Hz}$. Complex frequencies were used with a small imaginary part of the form $\operatorname{Im}(\omega)=-0.7 \Delta \omega$ to avoid the aliasing phenomena. The effect of the complex frequency produces a damped response with a time decay of the form $e^{-0.7 \Delta \omega t}$. The effect of the imaginary part would remove in the time response applying an exponential window $e^{0.7 \Delta \omega t}$. 


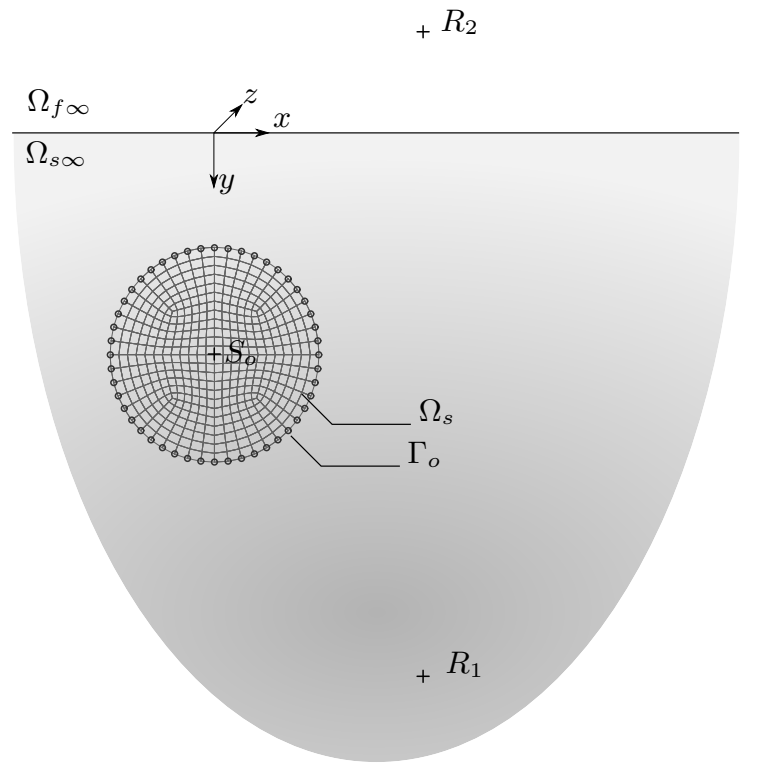

Figure 4: Problem discretisation (figure not scaled). Soil domain $\left(\Omega_{s \infty}\right)$, air above the ground surface $\left(\Omega_{f \infty}\right)$, solid enclosure $\left(\Omega_{s}\right)$, soil-structure interface $\left(\Gamma_{o}\right)$, source point $\left(S_{o}\right)$ and observation points $\left(R_{1}\right.$ and $\left.R_{2}\right)$.

The solid subdomain has circular geometry, centred at the source point $S_{o}$. The radius of the cylindrical inclusion was set to $r=0.05 \mathrm{~m}$ to verified the proposed formulation with a reliable problem that allowed fast computations without loss of validity. The limiting interface within the unbounded solid half-space $\left(\Gamma_{o}\right)$ was represented with the BEM, and the FEM was used to represent the enclosed region $\left(\Omega_{s}\right)$. The problem solution is defined by Equation (29). The number of boundary elements used to discretise the solid interface was 48 , enough to represent the wavelength of the shear waves and the geometry adequately. The solid subdomain was modelled with 480 solid finite elements. The ratio between the element size and the minimum wavelength was $7.2 \times 10^{-4}$. The limiting interface at the fluid-solid formation does not need to be discretised because the stated boundary conditions at the fluid-solid formation, defined by Equation (9) are implicitly satisfied in the fundamental solution.

First, the surface terms of the Green's function are analysed in order to set an appropriate wavenumber $k_{x}$ interval where Equation (10) converges. Figure 5 shows the Green's function terms $\widetilde{\mathcal{G}}_{x y}^{\text {surf }}, \widetilde{\mathcal{G}}_{y y}^{\text {surf }}$ and $\widetilde{\mathcal{G}}_{z y}^{\text {surf }}$ at receiver $R_{1}$, and the pressure field $\widetilde{\sigma}_{f y}$ at $R_{2}$, when the source is acting in the $y$ direction in $S_{o}$ at frequency $\omega=314.16 \mathrm{rad} / \mathrm{s}$. The pressure field $\widetilde{\sigma}_{f y}$ is given by the following expression [21]:

$$
\widetilde{\sigma}_{f y}=-\frac{i}{2 \pi}\left(\frac{E_{f}}{\nu_{x}^{f}} D^{y}\right)
$$

where $E_{f}=e^{-i \nu_{x}^{f} y}$, and $D^{y}=E_{b}^{i} \overline{\mathbf{D}}^{y}(1)+E_{c}^{i} \overline{\mathbf{D}}^{y}(2)$ is the fluid amplitude potential computed by Equation $(15)$.

Figure 5 shows the surface terms represented versus dimensionless wavenumbers $\bar{k}_{x}=k_{x} c_{s} / \omega$ and $\bar{k}_{z}=$ 
$k_{z} c_{s} / \omega$. Superimposed on this figure we can see the dispersion curve $\bar{k}_{R}=c_{s} / c_{R}$ of the Rayleigh waves with propagation velocity $c_{R}=2442 \mathrm{~m} / \mathrm{s}$, and the dispersion curve $\bar{k}_{F}=c_{s} / c_{f}$ of the dilatational pressure waves in the fluid. The dispersion curve $\bar{k}_{C}=c_{s} / C$ of waves with phase velocity $C$ is also represented. The phase wave velocity was estimated as:

$$
C(\omega)=\frac{\omega}{\sqrt{k_{x}^{* 2}(\omega)+k_{z}^{* 2}(\omega)}}
$$

where $k_{x}^{*}$ and $k_{z}^{*}$ denotes the wavenumbers where $\widetilde{\mathcal{G}}_{y y}^{\text {surf }}\left(k_{x}, y, k_{z}, \omega ; \widehat{\mathbf{x}}_{0}\right)$ takes its maximum value.

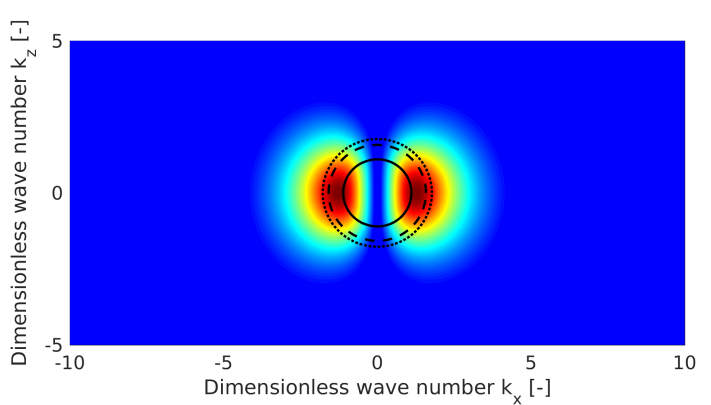

(a)

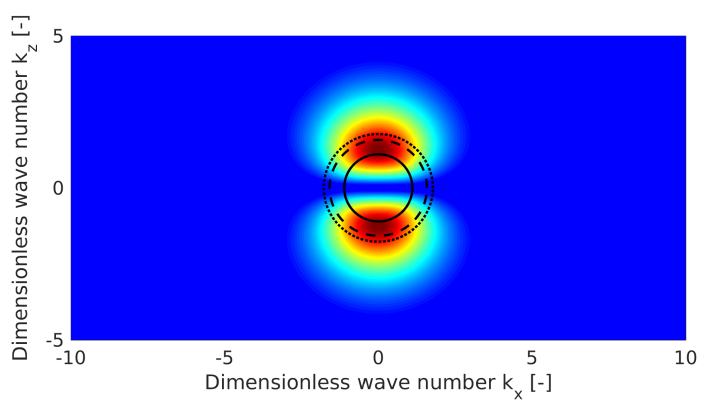

(c)

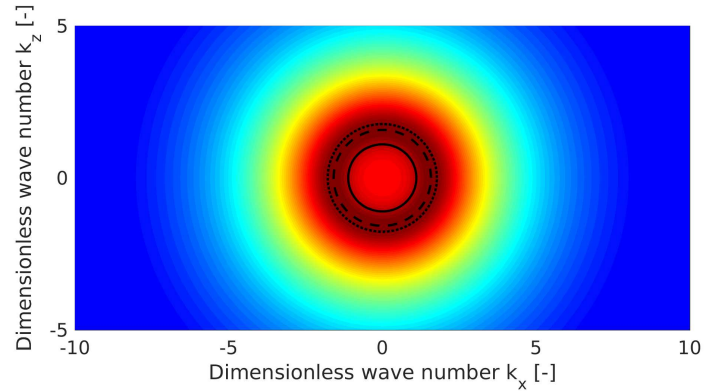

(b)

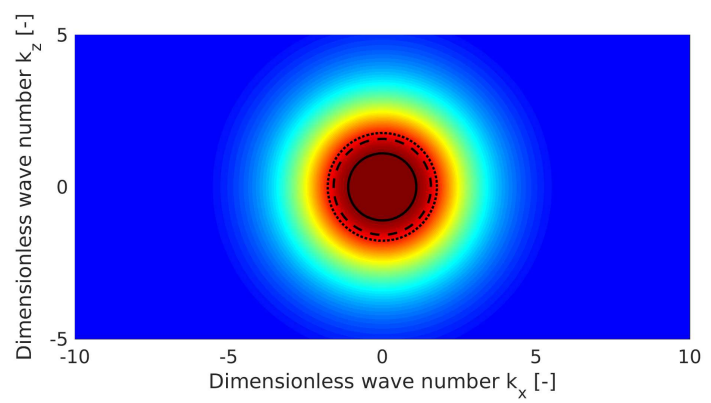

(d)

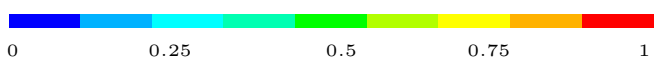

Figure 5: Real part of the Green's function for displacements (a) $\widetilde{\mathcal{G}}_{x y}^{\text {surf }}\left(\times 10^{14} \mathrm{~m}\right)$, (b) $\widetilde{\mathcal{G}}_{y y}^{\text {surf }}\left(\times 10^{14} \mathrm{~m}\right)$, and (c) $\widetilde{\mathcal{G}}_{z y}^{\text {surf }}$ $\left(\times 10^{14} \mathrm{~m}\right)$ at receiver $R_{1}$, and pressure $(\mathrm{d}) \widetilde{\sigma}_{f y}\left(\times 10^{5} \mathrm{~Pa}\right)$ at receiver $R_{2}$. Dimensionless wavenumber $\bar{k}_{R}$ (solid line), $\bar{k}_{C}$ (dashed line), and $\bar{k}_{F}$ (dotted line) are also represented.

The aforementioned dispersion curves define circumferential geometric regions where surface terms reach local maxima. Afterwards the Green's function varies smoothly and decays to zero value. In this work, the surface terms $\widetilde{\mathcal{G}}^{\text {surf }}$ were computed using 500 logarithmic spaced points for $\bar{k}_{x}$, from $10^{-6}$ to $10^{6}$, to ensure the convergence of Equation (10).

Figure 6 compares the analytical solution and the numerical results for solid displacement at receiver $R_{1}$, and the pressure field at receiver $R_{2}$. A constant wavenumber $k_{z}=0.4 \mathrm{rad} / \mathrm{m}$ was assumed for the verification 
of the proposed formulation. Numerical results are in good agreement with the reference solution [21].

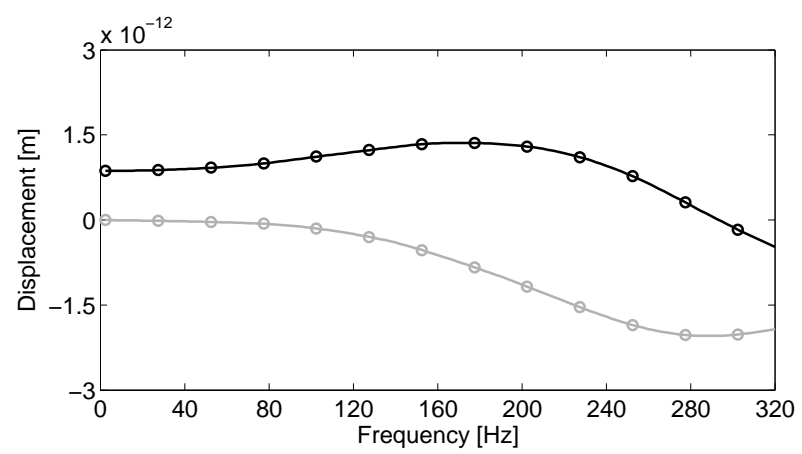

(a)

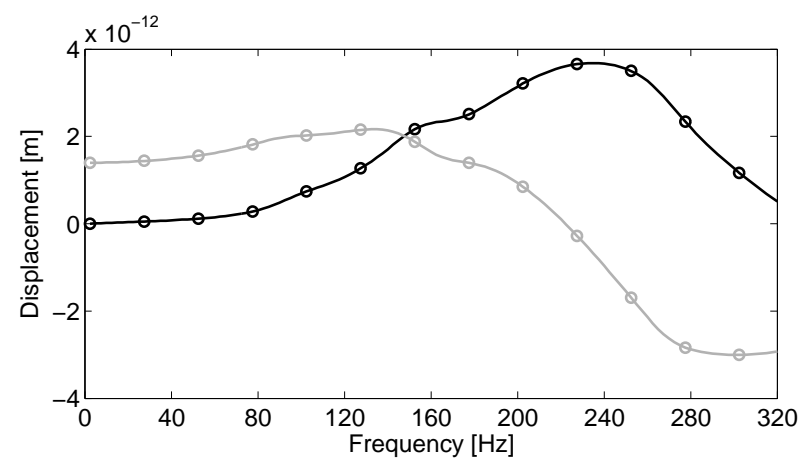

(c)

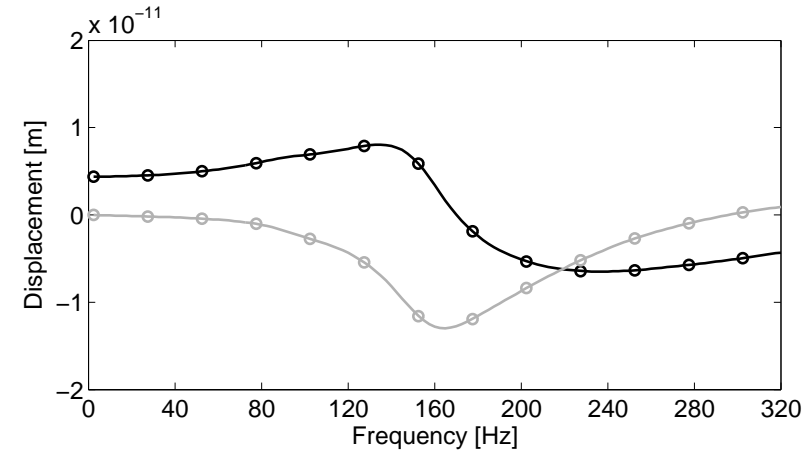

(b)

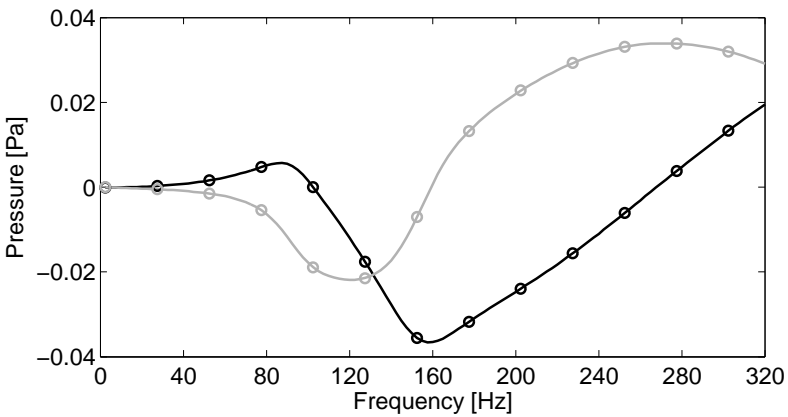

(d)

Figure 6: Real (black) and imaginary (grey) parts of the analytical solution [21] (solid lines) and numerical results (circles) for (a) horizontal, (b) vertical, and (c) longitudinal displacements at receiver $R_{1}$, and (d) pressure at receiver $R_{2}$, due to a vertical load acting at $S_{o}$.

The 3D problem solution is given by Equation (1) as the superposition of $2 \mathrm{D}$ problems with different wavenumbers $k_{z}$. This equation converges and can be solved by a finite sum of terms. In order to study the convergence, Figure 7 shows the problem solution at $R_{1}$ and $R_{2}$ for a range of frequencies and wavenumbers $\bar{k}_{z}$, and the previous dispersion curves were also represented. The phase wave $\bar{k}_{C}$ and the Rayleigh wave $\bar{k}_{R}$ dispersion curves intersect each other at the frequency $f_{c o}=118 \mathrm{~Hz}$. Local maxima were found around $\bar{k}_{C}$ at lower frequencies, after which the solution decays for wavenumbers higher than $\bar{k}_{R}$. Additionally, the fluid pressure is mainly found around the region defined by $\bar{k}_{R}$ and $\bar{k}_{F}$, and becomes significant from the frequency. Thus, the convergence of Equation (1) would be ensured for wavenumbers $\bar{k}_{z}$ higher than $\bar{k}_{F}$ from the frequency $f_{c o}$. However, the computation for lower frequencies should be extended for a few wavenumbers until the solution decays to zero. 


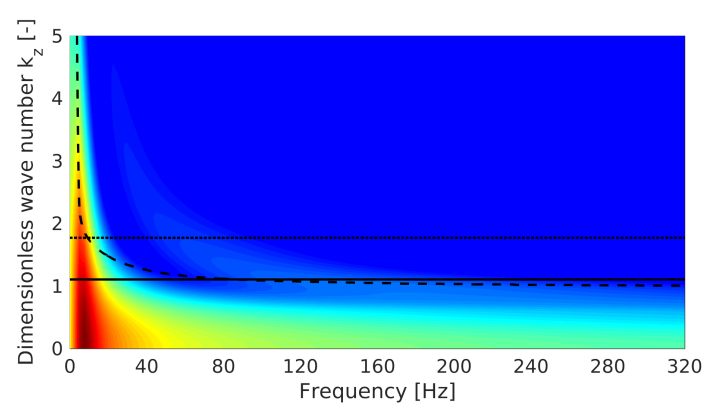

(a)

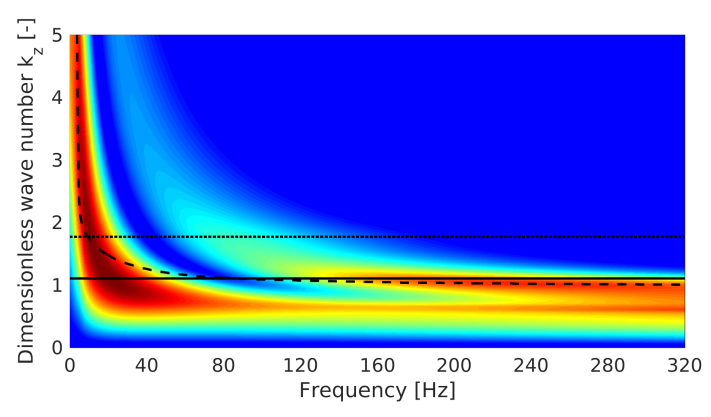

(c)

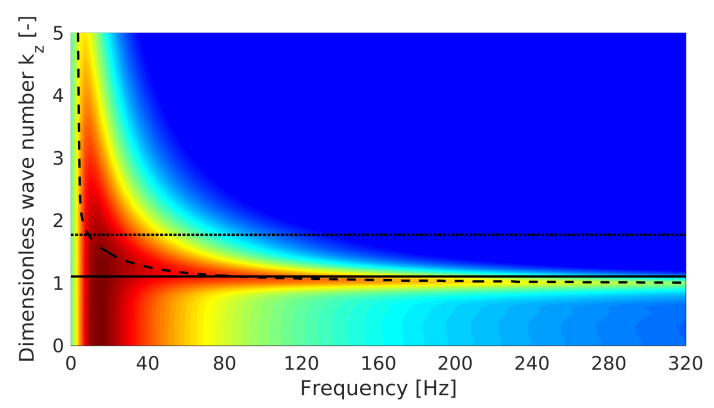

(b)

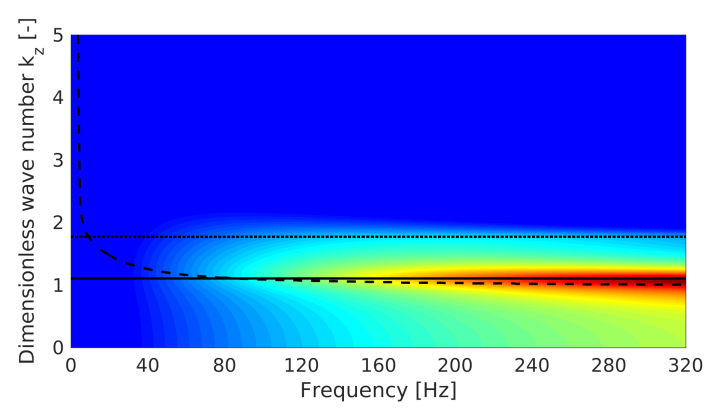

(d)

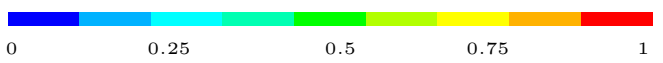

Figure 7: Amplitude of (a) horizontal $\left(\times 10^{12} \mathrm{~m}\right)$, (b) vertical $\left(\times 10^{11} \mathrm{~m}\right)$, and (c) longitudinal $\left(\times 10^{12} \mathrm{~m}\right)$ displacements at receiver $R_{1}$, and (d) pressure field $\left(\times 10^{2} \mathrm{~Pa}\right)$ at receiver $R_{2}$, due to a vertical load acting at $S_{o}$. Dimensionless wavenumber $\bar{k}_{R}$ (solid line), $\bar{k}_{C}$ (dashed line), and $\bar{k}_{F}$ (dotted line) are also represented.

\section{Applications}

This section explores ground vibrations and radiated noise in three applications. First, the wave field radiated by a single tunnel due to harmonic loads is evaluated. Next, the scattered wave field caused by the insertion of a second tunnel is studied in a twin tunnel system. Finally, changes in sound pressure at the free-field produced by the insertion of mitigation systems in the underground structure are assessed. In this last example, an underground railway station with an acoustic barrier is considered.

\subsection{Radiated noise and vibration generated by a tunnel}

This example analyses the noise and vibration field radiated from a tunnel embedded in a homogeneous half-space (Figure 8). Soil displacements and the sound pressure level inside the tunnel and above the ground surface due to a harmonic load $P=2 \pi \mathrm{N}$ are studied for a maximum frequency range of $f_{\max }=250 \mathrm{~Hz}$. The soil response of this problem has previously been studied by Gupta et al. [31]. This reference has been given to verify the conclusions obtained with the proposed methodology. 


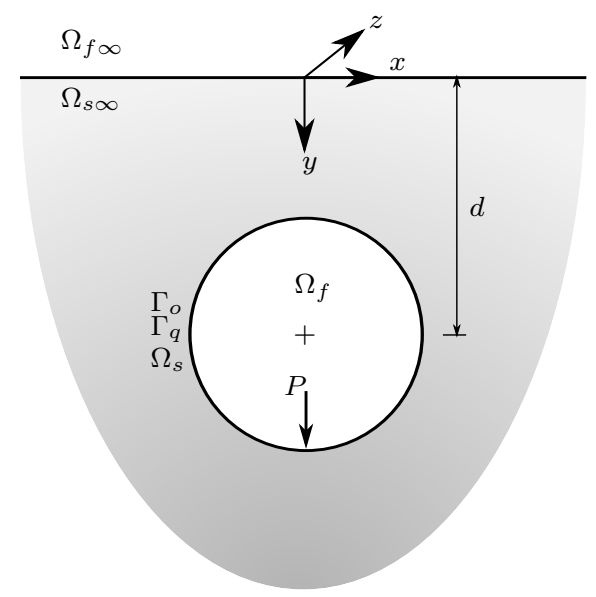

Figure 8: Definition of tunnel and subdomains (figure not scaled). Soil domain $\left(\Omega_{s \infty}\right)$, air above the ground surface $\left(\Omega_{f} \infty\right)$, solid $\left(\Omega_{s}\right)$ and fluid $\left(\Omega_{f}\right)$ enclosures, the fluid-structure $\left(\Gamma_{q}\right)$ and the soil-structure $\left(\Gamma_{o}\right)$ interfaces, tunnel depth $(d)$ and acting force $(P)$.

The tunnel is at depth $d=20 \mathrm{~m}$, has radius $r=3.0 \mathrm{~m}$ and wall thickness $t=0.3 \mathrm{~m}$. The lining has concrete properties with Young's modulus $E=35 \times 10^{9} \mathrm{~N} / \mathrm{m}^{2}$, Poisson's ratio $\nu=0.25$, and density $\rho=2500 \mathrm{~kg} / \mathrm{m}^{3}$. The soil has a P-wave propagation velocity $c_{p}=500 \mathrm{~m} / \mathrm{s}$, an S-wave propagation velocity $c_{s}=250 \mathrm{~m} / \mathrm{s}$, and a density $\rho_{s}=1750 \mathrm{~kg} / \mathrm{m}^{3}$. The sound propagation velocity is $c_{f}=340 \mathrm{~m} / \mathrm{s}$, and the air density takes a value of $\rho_{f}=1.22 \mathrm{~kg} / \mathrm{m}^{3}$.

The analysis was carried out in the frequency-wavenumber domain for frequencies ranging from $2 \mathrm{~Hz}$ to $250 \mathrm{~Hz}$, with a frequency step of $2 \mathrm{~Hz}$. The longitudinal wavenumber varies from $\bar{k}_{z}=0$ to $\bar{k}_{z}=3 \times \bar{k}_{F}=4.08$, which ensure the radiated field is computed accurately at receivers far from the application load. The wavenumber step was set so as to adequately represent $n=100$ acoustic wavelengths, $\Delta \bar{k}_{z}=1 / n$.

The tunnel lining is represented with 216 shell finite elements $\left(\Omega_{s}\right)$, and the discretisation matches with the boundary element mesh used for the soil-structure interface $\left(\Gamma_{o}\right)$, as well as the fluid-structure interface $\left(\Gamma_{q}\right)$. The $2.5 \mathrm{D}$ shell element is a two-nodes line element with four degree of freedom at each node (three displacements and one rotation around the $z$ axis). The air volume inside the tunnel $\left(\Omega_{f}\right)$ is represented with 9562 fluid finite elements $\underline{\underline{20}]}$, allowing the representation of wavelengths with at least 6 elements.

Figure 9 shows vertical displacements and sound pressure levels at three observation points on the ground surface $(y=0 \mathrm{~m})$, at different distances from the tunnel axis: $x=0 \mathrm{~m}, x=4 \mathrm{~m}$, and $x=16 \mathrm{~m}$. The frequency content has an undulating behaviour because different waves are propagating through the fluid-solid formation, and the frequency step between subsequent undulations becomes lower as the distance of the observation point increases, but remains uniform in the frequency range. The Green's function for the fluid-solid formation is also represented in this figure to show that differences between the field radiated by the tunnel and the fluid-solid formation behaviour are due to the waves scattered by the structure. The 
mismatch becomes more significant as fluid-solid wavelengths are smaller than the tunnel size. These results are consistent with those reported in Reference [31] for the soil response.

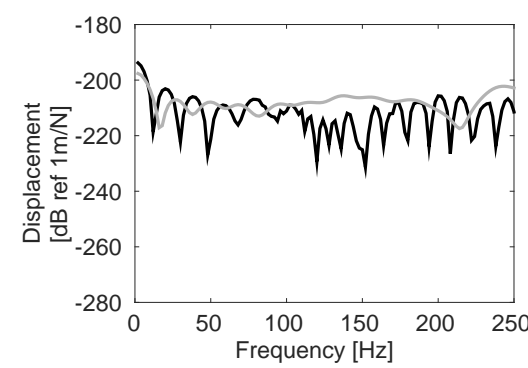

(a)

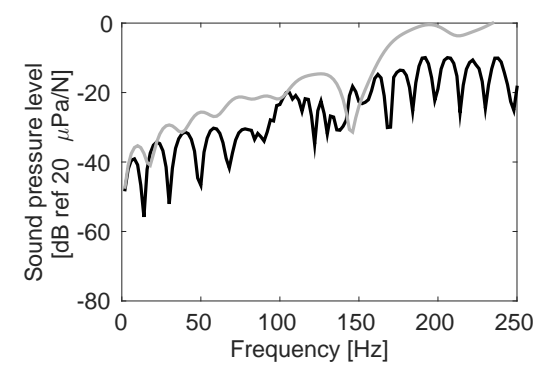

(d)

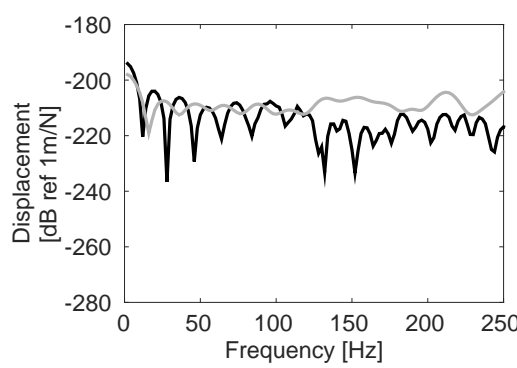

(b)

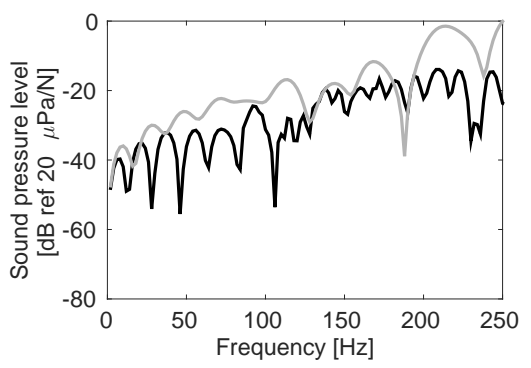

(e)

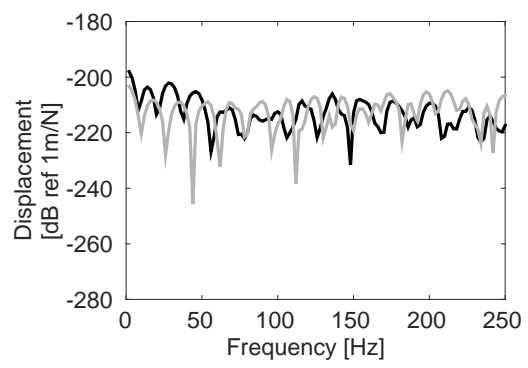

(c)

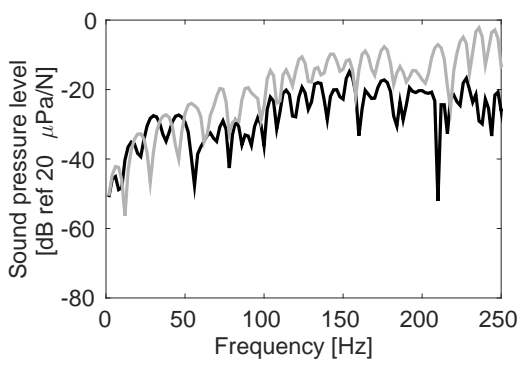

(f)

Figure 9: (a-c) Vertical displacement (black line), and (d-f) sound pressure level (black line), at points on the ground surface with $(\mathrm{a}, \mathrm{d}) x=0 \mathrm{~m},(\mathrm{~b}, \mathrm{e}) x=4 \mathrm{~m}$, and $(\mathrm{c}, \mathrm{f}) x=16 \mathrm{~m}$ due to a load acting at the tunnel invert. The analytical solution for a homogeneous fluid-solid formation [21] is also represented (grey line).

Vertical soil displacement and sound pressure level distribution, both inside the tunnel and above the ground surface, are represented in Figure 10 for frequencies of $10 \mathrm{~Hz}$ and $80 \mathrm{~Hz}$. Results were computed at a grid of 7141 receivers equally spaced, and the 3D solution was computed afterwards. Maximum displacements were found around the tunnel invert according to the soil wavelength. A shadowed part is observed above the tunnel where the response is lower. Moreover, the sound pressure distribution is defined by the fluidmomentum relation, which links fluid pressure with soil displacement. Therefore, radiated waves at the free field show a maximum above the tunnel, and the maximum inside the tunnel is found around the lining. Fluid wavelengths are longer than those in the solid, according to the wave propagation velocity in each medium.

\subsection{Scattered wave field by a twin tunnel system}

This example examines the wave field scattered by two tunnels embedded in a homogeneous half-space (Figure 11). One of these tunnels is subject to a dynamic force $P=2 \pi \mathrm{N}$ applied to the tunnel invert. The importance of the tunnel's interactions is analysed through the insertion gain that results from the existence 


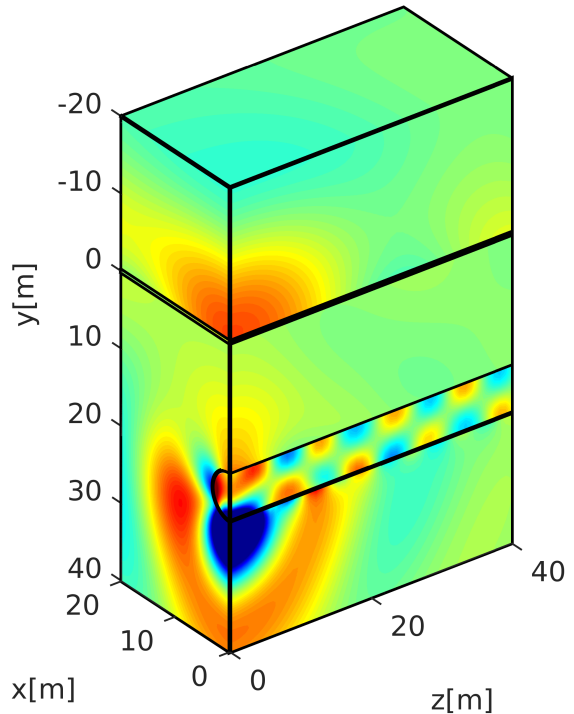

(a)

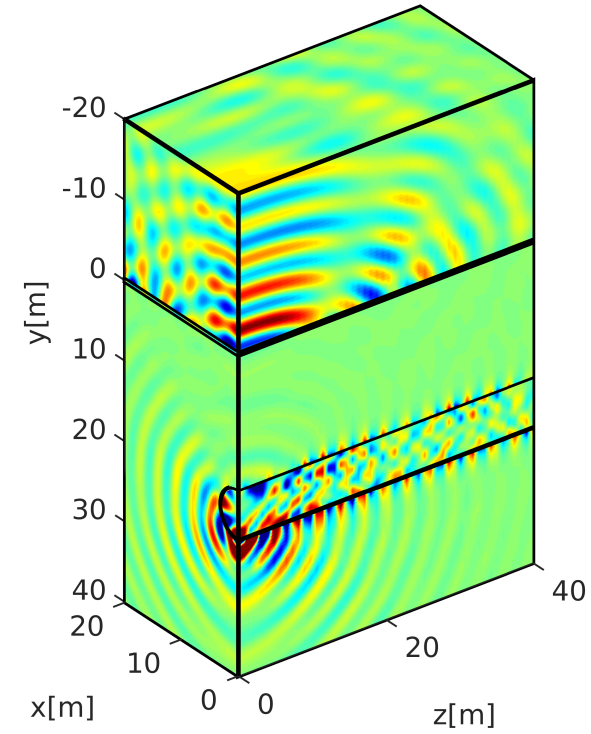

(b)

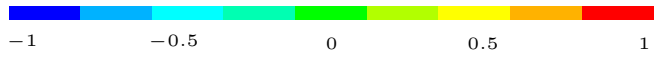

Figure 10: Real part of tunnel and soil vertical displacements $\left(\times 10^{7} \mathrm{~m}\right)$, and sound pressure inside the tunnel $\left(\times 10^{2} \mathrm{~Pa}\right.$ at $10 \mathrm{~Hz}$ and $\times 10^{1} \mathrm{~Pa}$ at $\left.80 \mathrm{~Hz}\right)$ and above ground surface $\left(\times 10^{3} \mathrm{~Pa}\right)$, produced by a point load of (a) $10 \mathrm{~Hz}$ and (b) $80 \mathrm{~Hz}$ acting on the tunnel invert.

of a second tunnel. Kuo et al. [32] studied the effect of a twin tunnel system on the wave propagation in an unbounded soil domain. The same system is analysed in this example. The radiated noise is also studied.

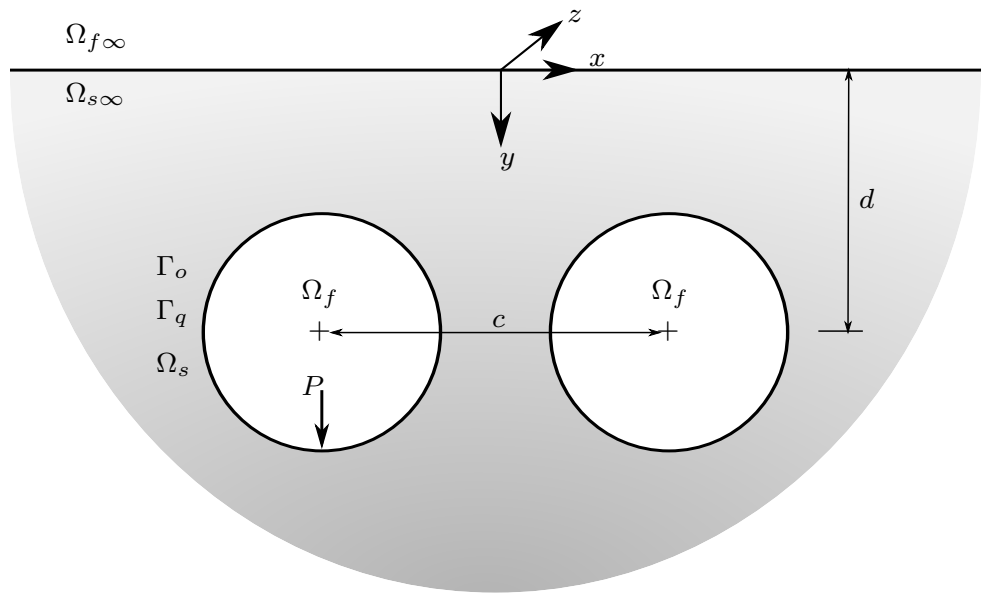

Figure 11: Diagram of twin tunnel (figure not scaled). Soil domain $\left(\Omega_{s \infty}\right)$, air above the ground surface $\left(\Omega_{f \infty}\right)$, solid $\left(\Omega_{s}\right)$ and fluid $\left(\Omega_{f}\right)$ enclosures, the fluid-structure $\left(\Gamma_{q}\right)$ and the soil-structure $\left(\Gamma_{o}\right)$ interfaces, tunnel depth $(d)$ and acting force $(P)$.

Both tunnels are located at depth $d=20 \mathrm{~m}$ and they are separated by a distance of $c=10 \mathrm{~m}$, they have radius $r=3.0 \mathrm{~m}$ and the wall thickness is $t=0.3 \mathrm{~m}$. The lining has the properties of concrete, with 
Young's modulus $E=35 \times 10^{9} \mathrm{~N} / \mathrm{m}^{2}$, Poisson's ratio $\nu=0.25$, and density $\rho=2500 \mathrm{~kg} / \mathrm{m}^{3}$. The soil has a P-wave propagation velocity $c_{p}=500 \mathrm{~m} / \mathrm{s}$, an S-wave propagation velocity $c_{s}=250 \mathrm{~m} / \mathrm{s}$, and a density $\rho_{s}=1750 \mathrm{~kg} / \mathrm{m}^{3}$. The sound propagation velocity is $c_{f}=340 \mathrm{~m} / \mathrm{s}$, and the air density takes a value of $\rho_{f}=1.22 \mathrm{~kg} / \mathrm{m}^{3}$.

The response of the twin-tunnel system is computed using the proposed methodology. Each tunnel is modelled with 56 shell elements and the volume of air within the tunnels is discretised with 588 fluid elements. The boundary element mesh matches the finite element discretisation. The solution is computed for a vertical load applied on the left-hand tunnel invert (Figure 11) acting at $60 \mathrm{~Hz}$. The wavenumber $\bar{k}_{z}$ range was set as in the previous example.

Figure 12.(a) shows the vertical soil displacements and the sound pressure inside tunnels. The soil response exhibits a non-symmetric distribution around the vertical axis of the loaded tunnel due to the wave field scattered by the right-hand tunnel. Maximum displacements occurred around the source point. The sound pressure pattern of both tunnels is defined by the soil displacement around them. The sound pressure reaches its highest value in the loaded tunnel.

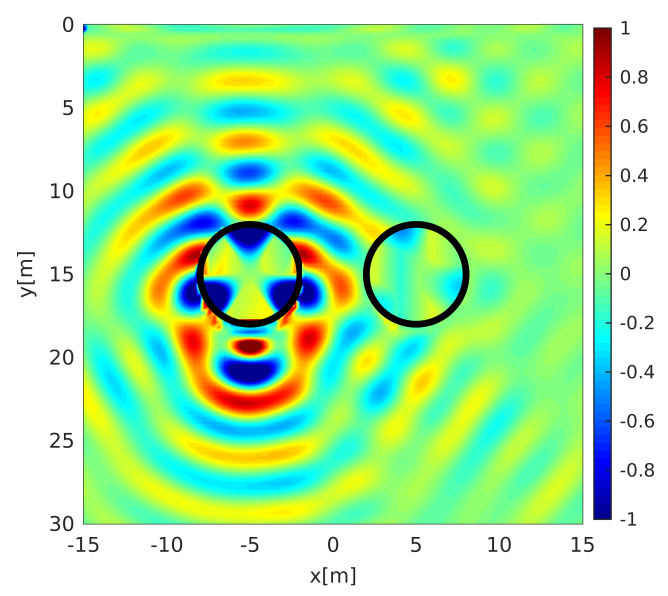

(a)

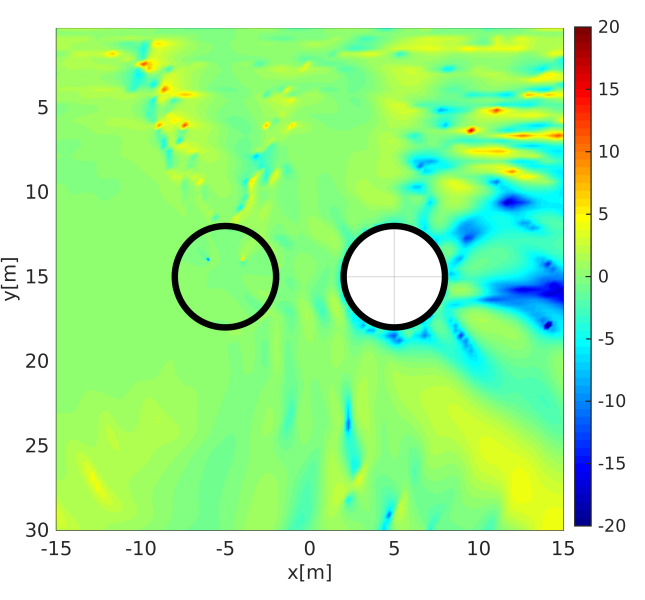

(b)

Figure 12: (a) Real part of vertical soil displacement $\left(\times 10^{7} \mathrm{~m}\right)$ and radiated noise inside the tunnels $\left(\times 10^{1} \mathrm{~Pa}\right)$, due to a harmonic load acting on the tunnel invert at $f=60 \mathrm{~Hz}$, and (b) the insertion gain from the response of a twin-tunnel system over that of a single-tunnel.

The effect of waves scattered by the right-hand tunnel is investigated in terms of the insertion loss in the single-tunnel and twin-tunnel systems. The second tunnel changes both the soil displacement distribution and the sound pressure inside the loaded tunnel, and this becomes more important at the right-hand tunnel, as can be seen in Figure 12.(b). These conclusions agree with those presented by Kuo et al. [32] for the ground response. In fact, the second tunnel modifies the sound pressure level distribution at the ground 
surface (Figure 13). The sound pressure field is distributed around the source point and comprises a circular region about $20 \mathrm{~m}$. The insertion loss also shows major differences between the tunnel systems.

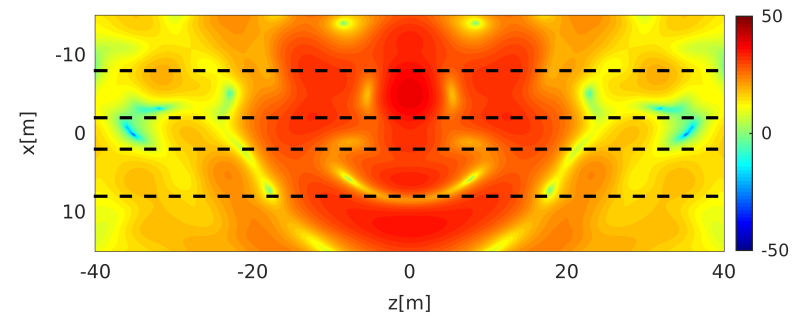

(a)

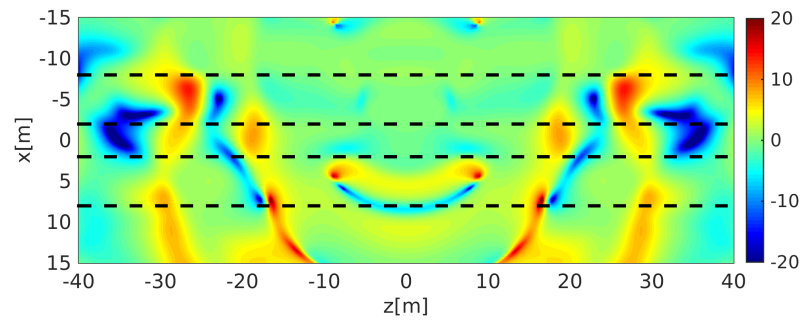

(b)

Figure 13: (a) Real part of sound pressure level [dB ref $20 \mu \mathrm{Pa}]$ at the ground surface due to a harmonic load acting on the tunnel invert at $f=60 \mathrm{~Hz}$, and (b) the insertion gain [dB] from the response of a twin-tunnel system over that of a single tunnel. Tunnel locations are marked by a dashed line.

\subsection{Noise radiated from an underground train station with acoustic insulation}

The last application examines the noise radiated by an underground railway station (Figure 14) that has an acoustic barrier that separates the two railway tracks. An acoustic point pressure load, located at $S_{0}(-2,14)$ simulates the noise of a passing train. This example was proposed by Tadeu et al. [19] for an unbounded domain to study the sound wave propagation inside the station in 2D cases. We have extended the problem to evaluate the influence of an acoustic barrier on the free-field radiated noise.

The station is at a depth $d=12 \mathrm{~m}$ and the geometry can be found in Figure 14. The structure has a concrete wall of thickness $t=0.3 \mathrm{~m}$, Young's modulus $E=50 \times 10^{9} \mathrm{~N} / \mathrm{m}^{2}$, Poisson's ratio $\nu=0.3$, and density $\rho=2500 \mathrm{~kg} / \mathrm{m}^{3}$. The soil has a P-wave propagation velocity $c_{p}=500 \mathrm{~m} / \mathrm{s}$, an $\mathrm{S}$-wave propagation velocity $c_{s}=250 \mathrm{~m} / \mathrm{s}$, and a density $\rho_{s}=1750 \mathrm{~kg} / \mathrm{m}^{3}$. The air takes sound propagation velocity $c_{f}=340 \mathrm{~m} / \mathrm{s}$, and density $\rho_{f}=1.22 \mathrm{~kg} / \mathrm{m}^{3}$. The acoustic barrier was assumed to be rigid, and $3.5 \mathrm{~m}$ long, and it was modelled as a discontinuity for the air volume $\Omega_{f}$. There is a gap of $1 \mathrm{~m}$ exists between the station invert and the barrier.

The vibration and radiated noise produced by a moving pressure load $P$ acting at point $S_{o}$ are analysed next. This load reproduces a moving source travelling in the $z$ direction at speed $c_{f}=\omega / k_{z}$. This speed is not a realistic traveling train speed but its interest is related with the wave propagation phenomenum in mediums with different wave propagation velocities. The source is assumed to be represented as a Ricker wavelet with a characteristic frequency of $145 \mathrm{~Hz}$ and amplitude $2 \pi \mathrm{Pa}$ [19]. The solution was computed for a frequency range from $2 \mathrm{~Hz}$ to $512 \mathrm{~Hz}$, with a frequency step of $2 \mathrm{~Hz}$. Complex frequencies with an imaginary part of the form $\operatorname{Im}(\omega)=-0.7 \Delta \omega$ were used to reduce the contribution of the virtual source to the response and to prevent the occurrence of aliasing phenomena. Each frequency step was solved for a wavenumber 


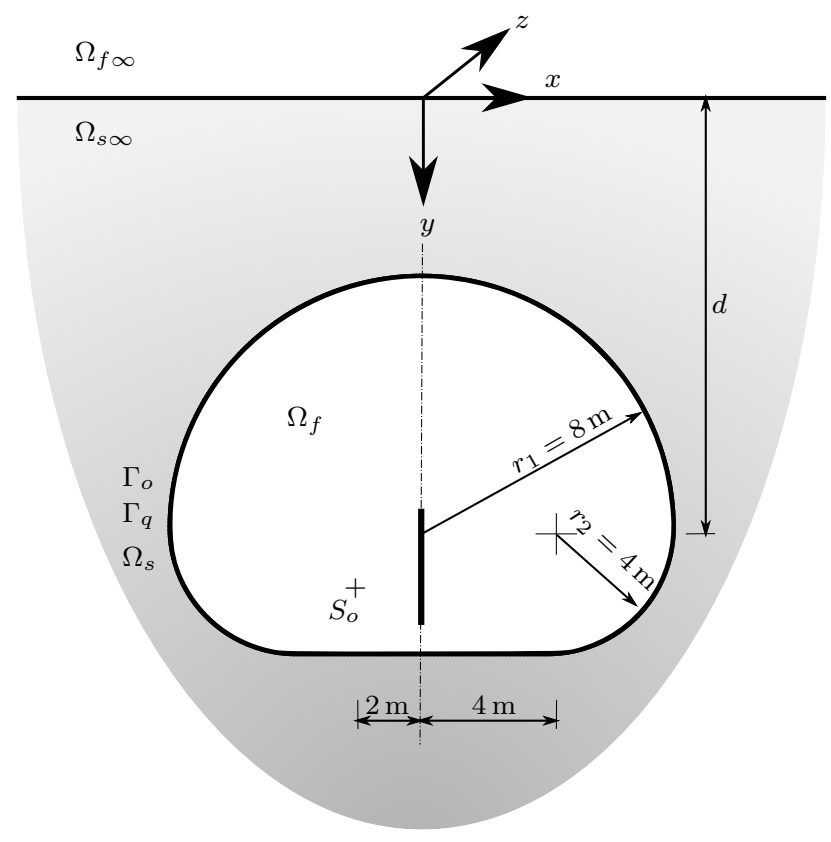

Figure 14: Diagram of underground station and definition of subdomains (figure not scaled). Soil domain $\left(\Omega_{s} \infty\right)$, air above the ground surface $\left(\Omega_{f \infty}\right)$, solid $\left(\Omega_{s}\right)$ and fluid $\left(\Omega_{f}\right)$ enclosures, the fluid-structure $\left(\Gamma_{q}\right)$ and the soil-structure $\left(\Gamma_{o}\right)$ interfaces, depth $(d)$ and source point $\left(S_{o}\right)$.

defined by the load speed. The characteristic element size enabled the minimum wavelength to be suitably represented with at least six elements.

Figure 15 compares the sound pressure distribution inside the station with and without the insulation barrier at the cross section defined by the coordinate plane $y=12 \mathrm{~m}$. The pressure distribution was represented for the time when the load passes at $z=0 \mathrm{~m}$. Time solutions were obtained by applying an inverse Fourier transform to the frequency response. The sound pressure fields exhibit a typical Mach cone and many differences between the two cases were found due to the presence of the barrier. The acoustic screen induced several wave reflections at the station wall and at the barrier.

The free-field radiated noise is due to ground-borne vibration defined by the stated boundary conditions at soil surface (Equation (9)). The sound pressure changes after the insertion of the acoustic barrier, as can be seen in Figure 16, and so does the soil displacement (Figure 17). The barrier redistributes displacements and tractions at the soil-structure interface and this modifies the wave field at the ground surface. The arrival time of $\mathrm{S}$ waves gives a spatial delay of $18 \mathrm{~m}$ in the displacements and sound pressure field.

\section{Conclusions}

This work has proposed a BEM-FEM formulation to study fluid and solid wave scattering in half-space formations. The method was formulated in $2.5 \mathrm{D}$ but is suitable for $3 \mathrm{D}$ problems whose material and 


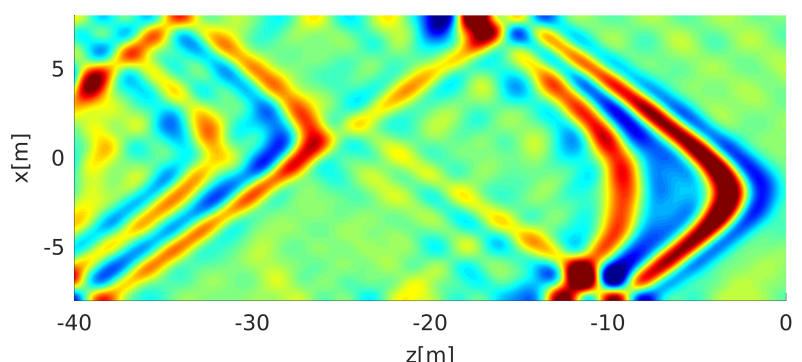

(a)

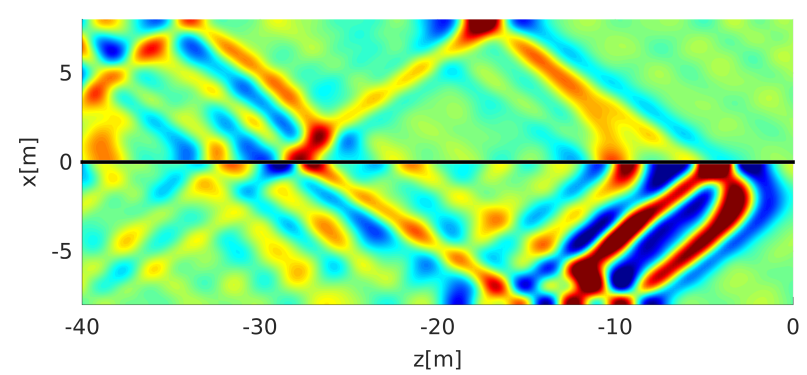

(b)

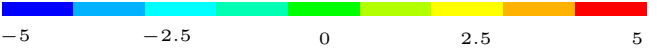

Figure 15: Radiated noise inside the station at a plane defined by the coordinate $y=12 \mathrm{~m}$, due to a moving load travelling at $v=c_{f}$, (a) without acoustic barrier, and (b) with acoustic barrier (black line).

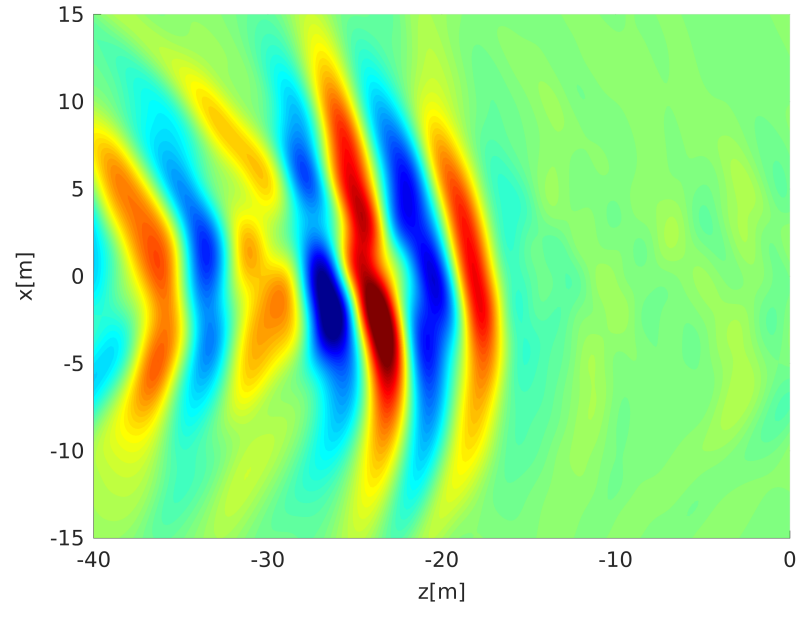

(a)

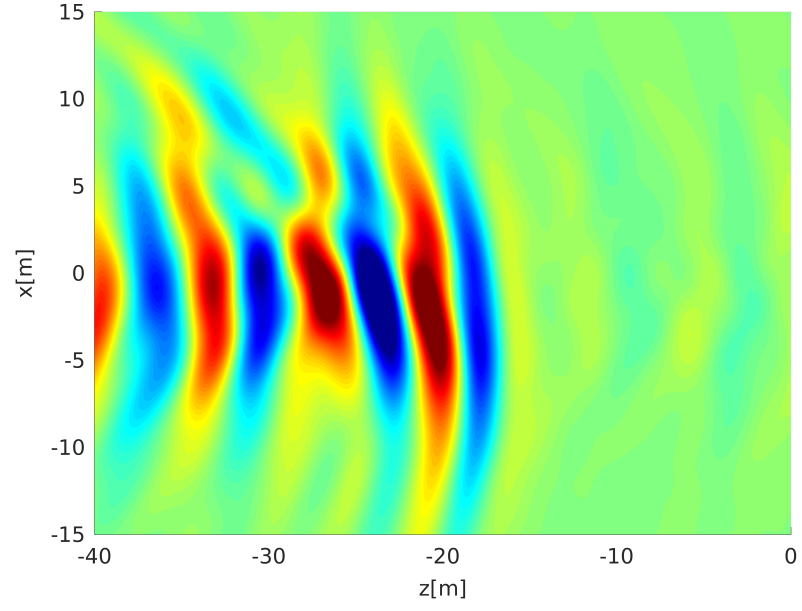

(b)

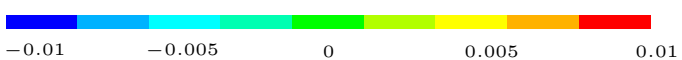

Figure 16: Radiated noise at the soil surface due to a moving load travelling at $v=c_{f}$ (a) without acoustic barrier, and (b) with acoustic barrier.

geometric properties are homogeneous in one direction. The proposed model has been developed to analyse the acoustic and elastic wave propagation from underground structures. The FEM modelling structures and enclosed fluid subdomains, while the BEM represents the soil-structure interface. The model was verified through a benchmark problem with a known analytical solution, and numerical results were in good agreement with the reference solution.

The main practical application of the method is the analysis of the noise and vibration radiated from tunnels, with a comprehensive formulation that predicts both sound pressure and soil displacement. Three examples were given that show the free-field radiated noise dependence on the scattered waves within the 


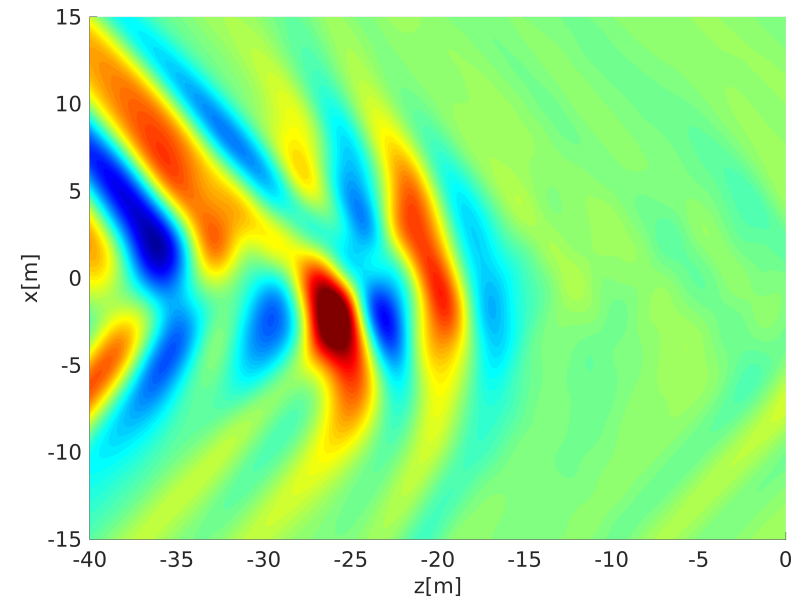

(a)

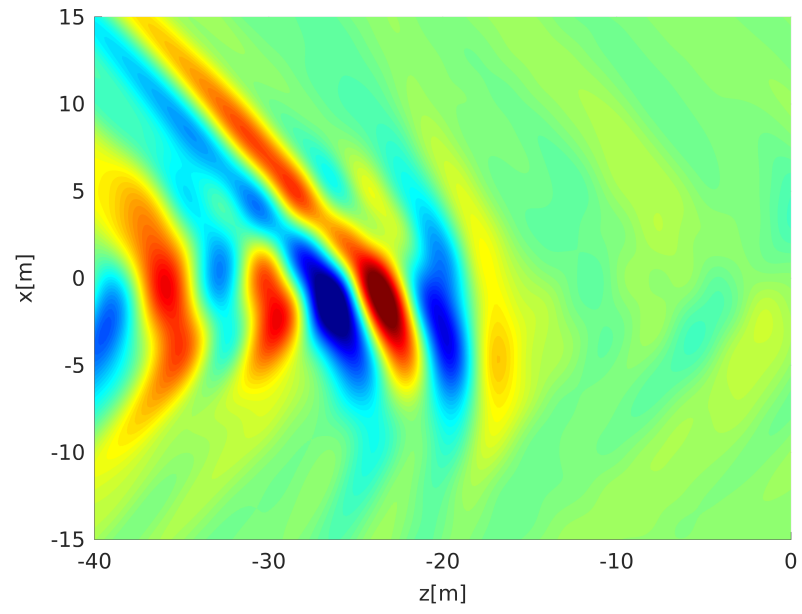

(b)

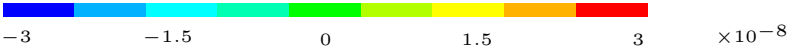

Figure 17: Vertical displacement at the soil surface, due to a moving load travelling at $v=c_{f}$, (a) without acoustic barrier, and (b) with acoustic barrier.

soil. The solution of the problem shows that:

- When a single tunnel is studied, the radiated wave field shows wavelengths according to soil and sound wave propagation velocity. A shadowed cone zone is found above the tunnel where soil displacements are lower. The wave field scattered by the tunnel is more important for wavelengths shorter than the tunnel size.

- If a twin-tunnel system is considered, the soil displacements and sound pressure field show a nonsymmetric distribution because of the waves scattered by the second tunnel. This effect changes the sound pressure level above the ground surface. However, the pressure field inside the loaded tunnel remains almost unchanged.

- Any modification of the pressure distribution inside structures involves a variation of both the pressure field at the ground surface and the soil displacements. This subject has been analysed in an underground station with an acoustic barrier, with the findings stated above.

These conclusions show that a fully coupled formulation could be advisable to accurately compute the noise and ground-borne vibration radiated from underground structures.

\section{Acknowledgments}

This work was framed within the POCI-01-0247-FEDER-003474 (coMMUTe) Project funded by Portugal 2020 through the Operational Programme for Competitiveness Factors (COMPETE 2020). This research 
was also funded by the Spanish Ministry of the Economy and Competitiveness (Ministerio de Economía y Competitividad) through research project BIA2013-43085-P. Financial support is gratefully acknowledged. The authors also wish to acknowledge the support provided by the Andalusian Scientific Computing Centre (CICA).

[1] A. Gidlöf-Gunnarsson, M. Ögren, T. Jerson, E. Öhrström, Railway noise annoyance and the importance of number of trains, ground vibration, and building situational factors, Noise and Health 14 (59) (2012) 190-201.

[2] H.V.C. Howarth, M.J. Griffin, The annoyance caused by simultaneous noise and vibration from railways, Journal of the Acoustical Society of America 89 (5) (1991) 2317-2323.

[3] E. Öhrström, A.-B. Skånberg, A field survey on effects of exposure to noise and vibration from railway traffic, part I: Annoyance and activity disturbance effects, Journal of Sound and Vibration 193 (1) (1996) 39-47.

[4] J.A. Sanz, M. Bonnet, J. Domínguez, Fast multipole method applied to 3-D frequency domain elastodynamics, Engineering Analysis with Boundary Elements 32 (10) (2008) 787-795.

[5] S. Chaillat, M. Bonnet, Recent advances on the fast multipole accelerated boundary element method for 3D time-harmonic elastodynamics, Wave Motion 50 (7) (2013) 1090-1104.

[6] Z.Y. Yan, X.W. Gao, The development of the pFFT accelerated BEM for 3-D acoustic scattering problems based on the Burton and Miller's integral formulation, Engineering Analysis with Boundary Elements 37 (2) (2013) 409-418.

[7] W. Hackbusch, Sparse matrix arithmetic based on H-matrices. Part I: Introduction to H-matrices, Computing (Vienna/New York) 62 (2) (1999) 89-108.

[8] W. Hackbusch, B. Khoromskij, A Sparse H-Matrix Arithmetic. Part: II: Application to Multi-Dimensional Problems, Computing (Vienna/New York) 64 (1) (2000) 21-47.

[9] D. Brunner, M. Junge, P. Rapp, M. Bebendorf, L. Gaul, Comparison of the fast multipole method with hierarchical matrices for the helmholtz-BEM, CMES - Computer Modeling in Engineering and Sciences 58 (2) (2010) 131-158.

[10] M. Messner, M. Schanz, An accelerated symmetric time-domain boundary element formulation for elasticity, Engineering Analysis with Boundary Elements 34 (11) (2010) 944-955.

[11] M. Bouchon, Discrete wave number representation of elastic wave field in three-space dimensions., Journal of Geophysical Research 84 (B7) (1979) 3609-3614.

[12] J.A. Forrest, H.E.M. Hunt, A three-dimensional tunnel model for calculation of train-induced ground vibration, Journal of Sound and Vibration 294 (4) (2006) 678-705.

[13] S. François, M. Schevenels, P. Galvín, G. Lombaert, G. Degrande, A 2.5D coupled FE-BE methodology for the dynamic interaction between longitudinally invariant structures and a layered halfspace, Computer Methods in Applied Mechanics and Engineering 199 (23-24) (2010) 1536-1548.

[14] P. Galvín, S. François, M. Schevenels, E. Bongini, G. Degrande, G. Lombaert, A 2.5D coupled FE-BE model for the prediction of railway induced vibrations, Soil Dynamics and Earthquake Engineering 30 (12) (2010) 1500-1512.

[15] S. Gupta, M.F.M. Hussein, G. Degrande, H.E.M. Hunt, D. Clouteau, A comparison of two numerical models for the prediction of vibrations from underground railway traffic, Soil Dynamics and Earthquake Engineering 27 (7) (2007) 608624.

[16] P. Coulier, A. Dijckmans, S. François, G. Degrande, G. Lombaert, A spatial windowing technique to account for finite dimensions in 2.5d dynamic soil-structure interaction problems, Soil Dynamics and Earthquake Engineering 59 (2014) 51-67.

[17] A.B. Nagy, P. Fiala, F. Márki, F. Augusztinovicz, G. Degrande, S. Jacobs, D. Brassenx, Prediction of interior noise in buildings generated by underground rail traffic, Journal of Sound and Vibration 293 (3-5) (2006) 680-690.

[18] P. Fiala, S. Gupta, G. Degrande, F. Augusztinovicz, A numerical model for re-radiated noise in buildings from underground 
railways, Notes on Numerical Fluid Mechanics and Multidisciplinary Design 99 (2008) 115-121.

[19] A. Tadeu, J. António, I. Castro, Coupling the BEM/TBEM and the MFS for the numerical simulation of acoustic wave propagation, Engineering Analysis with Boundary Elements 34 (4) (2010) 405-416.

[20] A. Romero, A. Tadeu, P. Galvín, J. António, 2.5D coupled BEM-FEM used to model fluid and solid scattering wave, International Journal for Numerical Methods in Engineering 101 (2) (2015) 148-164.

[21] A. Tadeu, J. António, 2.5D Green's functions for elastodynamic problems in layered acoustic and elastic formations, CMES - Computer Modeling in Engineering and Sciences 2 (4) (2001) 477-495.

[22] J. Domínguez, Boundary elements in dynamics, Computational Mechanics Publications and Elsevier Aplied Science, Southampton, 1993.

[23] A.J.B. Tadeu, P.F.A. Santos, E. Kausel, Closed-form integration of singular terms for constant, linear and quadratic boundary elements. Part 1. SH wave propagation, Engineering Analysis with Boundary Elements 23 (8) (1999) 671-681.

[24] A.J.B. Tadeu, P.F.A. Santos, E. Kausel, Closed-form integration of singular terms for constant, linear and quadratic boundary elements. Part 2. SV-P wave propagation, Engineering Analysis with Boundary Elements 23 (9) (1999) 757 $\underline{768 .}$

[25] A.J.B. Tadeu, E. Kausel, Green's functions for two-and-a-half-dimensional elastodynamic problems, Journal of Engineering Mechanics 126 (10) (2000) 1093-1097.

[26] A. Tadeu, J. António, L. Godinho, Analytical evaluation of the acoustic behavior of multilayer walls when subjected to three-dimensional and moving 2.5-dimensional loads, Journal of Vibration and Acoustics, Transactions of the ASME $135(6)$.

[27] L.E. Kinsler, A.R. Frey, A.B. Coppens, J.V. Sanders, Fundamentals of Acoustics, John Wiley and Sons, New York, 1982.

[28] A. Craggs, A finite element model for acoustically lined small rooms, Journal of Sound and Vibration 108 (2) (1986) 327-337.

[29] L. Gavrić, Finite Element Computation of Dispersion Properties of Thin-Walled Waveguides, Journal of Sound and Vibration 173 (1) (1994) 113-124.

[30] L. Gavrić, Computation of propagative waves in free rail using a finite element technique, Journal of Sound and Vibration 185 (3) (1995) 531-543.

[31] S. Gupta, Y. Stanus, G. Lombaert, G. Degrande, Influence of tunnel and soil parameters on vibrations from underground railways, Journal of Sound and Vibration 327 (1-2) (2009) 70-91.

[32] K.A. Kuo, H.E.M. Hunt, M.F.M. Hussein, The effect of a twin tunnel on the propagation of ground-borne vibration from an underground railway, Journal of Sound and Vibration 330 (25) (2011) 6203-6222. 